\title{
$\mu$-Opioid Receptors on Distinct Neuronal Populations Mediate Different Aspects of Opioid Reward-Related Behaviors
}

\author{
Amie L. Severino, ${ }^{1, *}$ Nitish Mittal, ${ }^{2,3,{ }^{*}}$ Joshua K. Hakimian, ${ }^{1}$ Nathanial Velarde, ${ }^{1}$ Ani Minasyan, ${ }^{1}$ \\ Ralph Albert, ${ }^{1}$ Carlos Torres, ${ }^{1}$ Nicole Romaneschi, ${ }^{1}$ DCamille Johnston, ${ }^{1}$ Suchi Tiwari, ${ }^{1}$ DAlex S. Lee, ${ }^{1}$ \\ Anna M. Taylor, ${ }^{4}$ Claire Gavériaux-Ruff, ${ }^{6,7,8,9,10}{ }^{1}$ Brigitte L. Kieffer, ${ }^{11}$ Christopher J. Evans, ${ }^{1,5}$ \\ Catherine M. Cahill, ${ }^{1,5}$ and Wendy M. Walwyn ${ }^{1,5}$
}

\section{https://doi.org/10.1523/ENEURO.0146-20.2020}

\begin{abstract}
${ }^{1}$ Department of Psychiatry and Biobehavioral Sciences, UCLA Semel Institute for Neuroscience and Human Behavior, David Geffen School of Medicine, University of California Los Angeles, Los Angeles, CA 90095, ${ }^{2}$ Division of Pharmacology and Toxicology, College of Pharmacy, University of Texas at Austin, Austin, TX 7871, ${ }^{3}$ ZS Associates, San Mateo, CA 94402, ${ }^{4}$ Department of Pharmacology, University of Alberta, Edmonton, Alberta,T6G 2R3 Canada, ${ }^{5}$ UCLA Brain Research Institute, University of California Los Angeles, Los Angeles, CA 90095, ${ }^{6}$ Department of Translational Medicine and Neurogenetics, Institut de Génétique et de Biologie Moléculaire et Cellulaire, Illkirch, France, UM7104, ${ }^{7}$ Université de Strasbourg, Illkirch, France, $67081,{ }^{8}$ Centre National de la Recherche Scientifique, Unité Mixte de Recherche 7104, Illkirch, France, ${ }^{9}$ Institut National de la Santé et de la Recherche Médicale, Unité 964, IIIkirch, France, ${ }^{10}$ Ecole Supérieure de Biotechnologie de Strasbourg, Illkirch, France, CS10413, and ${ }^{11}$ Department of Psychiatry, Douglas Mental Health Institute, McGill University, Montreal, Quebec, QC H4H 1R3 Canada
\end{abstract}

\begin{abstract}
$\mu$-Opioid receptors (MORs) are densely expressed in different brain regions known to mediate reward. One such region is the striatum where MORs are densely expressed, yet the role of these MOR populations in modulating reward is relatively unknown. We have begun to address this question by using a series of genetically engineered mice based on the Cre recombinase/loxP system to selectively delete MORs from specific neurons enriched in the striatum: dopamine 1 (D1) receptors, D2 receptors, adenosine 2a (A2a) receptors, and choline acetyltransferase (ChAT). We first determined the effects of each deletion on opioid-induced locomotion, a striatal and dopamine-dependent behavior. We show that MOR deletion from D1 neurons reduced opioid (morphine and oxycodone)-induced hyperlocomotion, whereas deleting MORs from A2a neurons resulted in enhanced opioid-induced locomotion, and deleting MORs from D2 or ChAT neurons had no effect. We also present the effect of each deletion on opioid intravenous self-administration. We first assessed the acquisition of this behavior using remifentanil as the reinforcing opioid and found no effect of genotype. Mice were then transitioned to oxycodone as the reinforcer and maintained here for $9 \mathrm{~d}$. Again, no genotype effect was found. However, when mice underwent $3 \mathrm{~d}$ of extinction training, during which the drug was not delivered,
\end{abstract}

\section{Significance Statement}

$\mu$-Opioid receptors (MORs) mediate the effects of the commonly misused and prescribed opioids. These receptors are expressed in different neurons and pathways mediating reward. Although it is well known that $\mu$ receptors in the midbrain regulate dopamine release and are important in mediating reward, little is known of the role of other populations that are expressed in the different neurons of the striatum, a hub of many reward pathways. In this study, we deleted selective populations of these receptors that are enriched in the striatum and studied the effect of each deletion on reward-related behaviors. We found that each population plays a specific role in reward demonstrating a more complex role than previously thought of how these receptors mediate reward. 
but all cues remained as during the maintenance phase, drug-seeking behavior was enhanced when MORs were deleted from A2a or ChAT neurons. These findings show that these selective MOR populations play specific roles in reward-associated behaviors.

Key words: floxed MOR; hyperlocomotion; intravenous self-administration; morphine; $\mu$-opioid receptor; oxycodone

\section{Introduction}

$\mu$-Opioid receptors (MORs), the principal target of addictive analgesics are widely expressed in diverse brain regions associated with reward (for review, see Le Merrer et al., 2009). MORs are expressed on the GABAergic neurons that innervate the dopaminergic neurons of the ventral tegmental area (VTA) so are poised to enable dopamine release (Ben Hamida et al., 2017; Charbogne et al., 2017) an important mediator of rewarding behavior. MORs are also expressed in the striatum which controls movement and the formation of behavioral habits associated with reward. These two behaviors, reward and locomotion, are mediated by different dopaminergic signaling profiles in distinct neurons (Howe and Dombeck, 2016) and are often used to generate a profile of reward behavior in mice (Mitchell et al., 2005; Zhang and Kong, 2017).

MORs are widely expressed in the different neuronal populations and subregions of the striatum (Wang et al., 1996, 1997; Wang and Pickel, 1998; Miura et al., 2008; Cui et al., 2014). They are expressed on dopamine 1 (D1) receptor, D2, and adenosine 2a (A2a) subpopulations of medium spiny neurons (Cui et al., 2014; Oude Ophuis et al., 2014). They are also expressed on cholinergic interneurons (Ponterio et al., 2013) and on cortical or thalamic glutamatergic neurons innervating medium spiny neurons (for review, see Miura et al., 2008). Within these different neuronal populations, MORs are differentially expressed in striatal subregions. For example, they are expressed in the patches or striasomes where they colocalize with dynorphin-expressing D1 medium spiny neurons (Brimblecombe and Cragg, 2017)

Received April 13, 2020; accepted July 30, 2020; First published August 28, 2020.

The authors declare no competing financial interests. N.M. is an employee of ZS Associates, but his contribution to this work is independent of ZS Associates, and ZS Associates did not participate or sponsor this work in any form. W.M.W., C.J.E., and C.M.C. are in communication with Nektar Therapeutics; however, Nektar Therapeutics did not participate or sponsor this work in any form.

Author contributions: A.L.S., N.M., J.K.H., N.V., A.M., N.R., C.J., A.M.T., C.G.-R., B.L.K., C.J.E., C.M.C., and W.M.W. designed research; A.L.S., J.K.H., N.V., A.M., R.A., C.T., N.R., C.J., S.T., A.S.L., A.M.T., and W.M.W. performed research; A.L.S., N.M., J.K.H., A.M., S.T., A.S.L., A.M.T., C.M.C., and W.M.W. analyzed data; A.L.S., N.M., C.G.-R., B.L.K., C.J.E., C.M.C., and W.M.W. wrote the paper.

This work was supported by the National Institute on Drug Abuse Grant DA005010 (to A.L.S., J.K.H., B.L.K., C.J.E., C.M.C., W.M.W.) and by the Shirley and Stefan Hatos Foundation.

${ }^{\star}$ A.L.S. and N.M. contributed equally to this work.

Correspondence should be addressed to Wendy M. Walwyn at wwalwyn@ g.ucla.edu.

https://doi.org/10.1523/ENEURO.0146-20.2020

Copyright (C) 2020 Severino et al.

This is an open-access article distributed under the terms of the Creative Commons Attribution 4.0 International license, which permits unrestricted use, distribution and reproduction in any medium provided that the original work is properly attributed. but also in the matrix where their expression is less and on D1 or D2 medium spiny neurons (Cui et al., 2014).

Although we do not fully understand the functional role of each of the striatal neuronal populations, we do have insight as to their function from their cellular expression patterns and electrophysiology studies. MORs are expressed presynaptically on glutamatergic afferents projecting to the striatum and postsynaptically on striatal dendrites and dendritic spines (Wang et al., 1996). Activation of these receptors inhibits both glutamatergic afferent activity and that of GABAergic collaterals from medium spiny neurons (Blomeley and Bracci, 2011; Ma et al., 2012; James et al., 2013). In addition, MORs inhibit cholinergic interneurons so regulating local spontaneous dopamine release (Sandor et al., 1992; Ponterio et al., 2013; Ponterio et al., 2018). Presynaptic MORs are also found on low threshold spike interneurons and so modulate their spontaneous activity (Elghaba and Bracci, 2017). At the behavioral level, several studies point toward a role of striatal MOR populations in reward behaviors. Earlier studies showed that ablating MOR-enriched striosomes of the dorsal striatum produces deficits in motorskill learning (Lawhorn et al., 2009). Forebrain MORs are known to play a role in alcohol, food and heroin reward behaviors (Ben Hamida et al., 2017; Charbogne et al., 2017) and in the hedonic reward value of food reward (Boulos et al., 2019). In addition, MOR re-expression on dynorphin expressing medium spiny neurons in an otherwise null background are sufficient to reinstate some, but not all, opioid reward behaviors (Cui et al., 2014).

Given the broad but diverse distribution of MORs on different neuronal subtypes throughout the striatum, we set out to determine the contribution of these MORs to opioid reward behaviors. In order to do this, we bred fIMOR mice, in which exons two and three of the MOR gene (oprm1) are flanked by LoxP, with four different Cre-recombinase mice (D1cre, D2cre, A2acre, ChATcre). We first verified these deletions using RNAScope in situ mRNA hybridization and quantitative PCR. We then assessed opioid-induced hyperlocomotor, sensitization of this effect, and intravenous opioid self-administration (IVSA). From these studies, we conclude that each of these MOR-expressing populations are required for distinct aspects of opioid reward-related behaviors.

\section{Materials and Methods}

\section{Experimental design \\ Subjects}

All procedures were authorized by the Institutional Animal Care and Use Committee (IACUC) and are in compliance with the Policies on the Use of Animals in Research as outlined by this journal. All transgenic mice 
used in this study were bred by the Animal Breeding Colony. D1fIMORs, D2fIMORS, A2aflMORs and choline acetyltransferase (ChAT) fIMORs were generated by breeding fIMOR mice (loxP sites flanking exons 2-3 of the oprm1 gene on a 50:50 C57BL/6J:129Sv background, stock \#030074, The Jackson Laboratory) with four Cre driver lines to obtain Cre recombinase on one (D1cre; stock \#030989UCD, D2cre; 032108-UCD, A2acre; 036158-UCD, MMRRC, $\mathrm{NIH}, \mathrm{DHHS}, 100 \%$ C57BL/6J) or two (ChAT-IRES-Cre; stock \#028861, The Jackson Laboratory, 100\% C57BL/6J) alleles and fIMOR on both alleles. Control fIMOR mice of the same background were generated as littermates from the breeding strategies used. Mice lacking all MORs (stock \#007559, 100\% C57BL/6J, The Jackson Laboratory) were bred as heterozygous pairs to generate knock-out (KO) and wild-type (WT) littermates. Male and female transgenic mice were used between age 8-32 weeks and 20-36 g of body weight. Animals were maintained on a 12/12 h light/dark cycle with ad libitum access to food and water, and experiments were conducted at ZT4-ZT8 (Zeitgeber Time). All mice were group housed for the duration of the experiment except for the IVSA experiments during which mice were singly housed in an enriched environment after surgery.

\section{Compounds}

All Schedule II drugs, remifentanil, oxycodone, cocaine, and morphine, were obtained from the NIDA Drug Supply Program (RTI).

\section{$R N A$ in situ hybridization and light sheet fluorescent} microscopy

Mice were euthanized, their brains removed and flash frozen. All equipment and surfaces were cleaned with RNase inhibitor solution and ISH (Advanced Cell Diagnostics) performed as previously described (Severino et al., 2018). To characterize MOR knock-down in the D1-, D2-, A2a-, and -fIMOR mice, the following riboprobes were used; oprm1 (catalog \#315841, Atto 550), drd2 (catalog \#406501-C2, Alexa Fluor 488), and drd1a (catalog \#406491-C3, Atto 647). To characterize MOR knock-down in ChATfIMOR mice, the same oprm1 and drd1a riboprobes were used as well as a ChAT riboprobe (catalog \#408731-C2, Alexa Fluor 488). RNA in situ hybridization was imaged using a $63 \times$ oil immersion objective on a Leica SP8 stimulated emission depletion microscope (STED, Leica Microsystems) at the Advanced Light Microscopy Core. The images were compiled in Adobe Illustrator 2019 and brightness and contrast and the tonal adjustments feature uniformly applied across the entire composite image. To determine the extent to which MOR was deleted from specific neuronal types within each of the mouse lines generated, we counted the number of drd1a, drd2, or ChAT-positive cells and then determined the number of these cells that were MOR positive (having a minimum of three grains). The data are expressed as the percentage of MOR-positive cells within each of the subgroups (drd1a, drd2, or ChAT).

\section{Quantitative reverse transcription- $P C R$ ( $q P C R$ )}

qPCR was performed in flMOR, D1-, D2-, A2a-, and ChAT-fIMOR mice to define the relative expression levels of
Table 1: The primer sequences used in the qPCR validation of MOR knock-down

\begin{tabular}{lc}
\hline & Gene primer sequences (5'-3') \\
\hline OPRM1 FWD & TCAAGGCCCTGGATTCCGTACCC \\
OPRM1 RVS & CGGGCAGACCAATGGCAGAAGAGA \\
DRD1 FWD & CTTGTCTGTGCCGCTGTCATCAGG \\
DRD1 RVS & GGCATGACCAAGACAGCCACCAAG \\
DRD2 FWD & TTGTTCTTGGTGTGTTCATC \\
DRD2 RVS & TATAGATGATGGGGTTCACG \\
ACTB FWD & TGTGCACTITATTGGTCTC \\
ACTB RVS & GATGTATGAAGGCTTTGGTC \\
\hline
\end{tabular}

OPRM1; $\mu$-opioid receptor, DRD1; dopamine 1 receptor, DRD2; dopamine 2 receptor, ACTB; $\beta$-actin control, FWD; forward, RVS; reverse.

oprm1, drd1a, and drd2 using the primers shown in Table 1 and methodology as previously described (Hakimian et al., 2017). Relative ratios comparing conditional KOs to fIMOR expression for each gene of interest were calculated by using $\beta$-actin as reference gene and the $2^{-\Delta \Delta \mathrm{Ct}}$ method to evaluate differential expression levels.

\section{Open-field locomotion}

Fiberglass open field boxes $(28 \times 28 \times 18 \mathrm{~cm})$ were placed on a horizontal glass pane $71 \mathrm{~cm}$ above an infrared camera (acA1300-60gm Basler ace camera) at 250 lux. After $2 \mathrm{~d}$ of habituation, mice were placed in the chamber for $15 \mathrm{~min}$ followed by a subcutaneous injection of saline or drug and placed back in the chamber for $60 \mathrm{~min}$ and their locomotion activity recorded (Ethovision XT10, Noldus). This was repeated at the same time of day for three consecutive days.

\section{IVSA}

An intravenous catheter $(0.2 \mathrm{~mm}$ i.d., $0.4 \mathrm{~mm}$ o.d., Norfolk Access) was inserted into the right jugular vein of mice under sterile conditions as previously described (James et al., 2013; Storey et al., 2016; Mittal et al., 2017). After $3 \mathrm{~d}$ of recovery, the mice began daily self-administration in operant chambers (Med-Associates) for $2 \mathrm{~h}$ or 50 reinforcers, whichever came sooner. A two-lever design was used in which the active cue and drug-paired lever, or the inactive lever, was randomly assigned. An active lever press resulted in an intravenous drug infusion $(0.67 \mu \mathrm{l} / \mathrm{g}$ body weight) and the presentation of a 10-s tone and visual light cue. Each reinforcer was followed by a 10-s "timeout" period during which no reinforcers could be delivered but presses could be made on either lever. On the first $2 \mathrm{~d}$ of this protocol, mouse exploration of the levers was facilitated by placing a drop of $20 \%$ sweetened condensed milk on both the active and inactive levers $(3 \times$ per session). The mice initially underwent $3-5 \mathrm{~d}$ of acquisition training using remifentanil $(0.05 \mathrm{mg} / \mathrm{kg} / \mathrm{infusion})$ at a fixed ratio of one (one lever press resulted in one infusion, FR1). Oxycodone $(0.25 \mathrm{mg} / \mathrm{kg} / \mathrm{infusion})$ was then used as the reinforcer for nine consecutive days, the maintenance phase, on the same FR1 schedule. This was followed by extinction training over $3 \mathrm{~d}$ during which the mice underwent the same FR1 schedule to a maximum of 50 reinforcers or $2 \mathrm{~h}$, but saline was delivered through the catheter. Catheter patency was tested using an infusion of propofol ( $20 \mu$ l of $1 \%$ propofol w/v in saline) every $5 d$. 


\section{Statistical analysis}

Power analyses of prior data indicate that power is 0.8 or greater with cell means of $n=8$ ( $n=12$ used for experiments where animal drop-out rates are expected because of jugular cannula failure, etc.). For experiments where we lacked sufficient prior data for an a priori power analysis, we used prior experience with similar methods to guide us. Although we did use male and female mice, we did not analyze sex as a biological factor as we did not have sufficient power to do so. All experiments included both genotypes with males and females representing $46 \%$ and $53 \%$, respectively, of the total number of mice used.

\section{Several analytical methods were used ANOVA}

One-way or two-way ANOVA was used to analyze data obtained from the RNA ISH, QPCR, total locomotion and the intrasession IVSA datasets using Prizm v8 (GraphPad) with further details provided in the results and statistical tables.

\section{Linear mixed models (LMM)}

LMM were used to analyze the intrasession locomotion data so as to examine the slope and so rate of change over time of this dataset. We also used LMM with coefficients accounting for random slope or intercept within subjects to define and interpret the intersession IVSA datasets. We used the ImerTest (Kuznetsova et al., 2017) package in $\mathrm{R}$ to run $\mathrm{LMM}$. The linear models were used to assess the effect of time, treatment group, or an interaction of these factors on each variable. The resulting model is a regression equation where the intercept or the slope is allowed to vary for each subject:

$$
Y_{\text {Characteristic }}=\beta_{0}+\beta_{\text {Group }} X_{\text {Group }} * \beta_{\text {Day }} X_{\text {Day }}+U_{\text {Subject }} \text {, }
$$

where $\mathrm{Y}_{\text {Characteristic }}$ is the characteristic being modeled (e.g., distance traveled, lever presses, etc.), each predictor variable is represented by its subscripted $X, U_{\text {subject }}$ represents the random intercept or slope associated with each individual subject. The coefficients $(\beta)$ are estimated and assessed for significance. Whenever a significant effect was observed, an ANOVA against a reduced null model was used to assess the impact of the respective factor.

\section{Results}

\section{Validation of the selectivity and extent of MOR knock- down in striatal subpopulations}

We first defined the selectivity of the loxP/Cre recombinase system by RNA in situ hybridization to examine cellspecific knock-down of the MOR encoding gene (oprm1) in the dorsolateral striatum. We found that, for cells labeled with the $d r d 1$ probe, oprm1 and $d r d 1$ colocalization was reduced in D1fIMORs (representative image, Fig. 1 Ai; quantified expression, Fig. $1 B i ; p<0.001$, Table 2, item a) and enhanced in D2fIMORs (Fig. 1Bi; $p<0.05$, Table 2, item a). For cells labeled by the drd2 probe, oprm1 and drd2 colocalization was reduced in D2flMORs (representative image, Fig. 1Ai; quantified expression, Fig. 1Bii; $p<0.01$, Table 2, item b). A2aflMORs showed oprm1 expression in drd1 + cells and a deletion from some, but not all $d r d 2+$ cells (representative image, Fig. $1 A i$; quantified expression, Fig. 1Bii; N.S, Table 2, item b). In assessing oprm1 expression in ChAT+ cells, we found a loss of oprm 1 expression in ChATfIMORs compared with fIMORs but no change in drd1 expression, a positive control (representative image, Fig. 1Aii; quantified expression, Fig. 1 Biii; $p<0.0001$, Table 2, item c).

qPCR was performed to determine overall striatal expression levels of oprm1, drd1, and drd2 in fIMOR in the conditional knock-down strains. We found a loss of oprm1 cDNA in D1fIMORs $(p=0.0001)$ and D2fIMORs $(p=0.015$; Fig. 1Ci; Table 2, item d) but no other line. There was no compensatory effect of these MOR deletions on drd1 (Fig. 1Cii; Table 2, item e) or drd2 (Fig. 1Ciii; Table 2, item f) expression in the different lines.

\section{Selective MOR deletions define specific roles of D1 and A2a MOR populations in opioid-induced hyperlocomotion \\ Oxycodone}

As the analgesic effects of oxycodone may be non-specific (Yang et al., 2016), we first examined the locomotor effect of oxycodone $(10 \mathrm{mg} / \mathrm{kg}$, s.c.) in mice lacking MORs in all cells, a global MOR KO, and their WT littermates, (Fig. 2A) over three consecutive days. On day 1 , we found no effect of oxycodone in MOR KOs compared with WTs $(p<0.01)$, a lack of effect that did not differ from WTs injected with saline $(p=0.92$, Table 3 , item a). By the third day, the oxycodone locomotor response had sensitized in WTs $(p<0.001)$ but no change was observed in KOs ( $p=0.97$, Table 3 , item b). The 5-min timebins of the intrasession data further show oxycodone-induced hyperlocomotion in WT but not KOs and sensitization of this response in only WTs over time (Fig. $2 B, p<0.01$; Table 3 , item c).

We then examined the dose-response relationship of oxycodone using 0 (saline), 1,3 , and $10 \mathrm{mg} / \mathrm{kg}$ subcutaneously in each of the genotypes (Fig. $3 A$ ). We found no effect of genotype following saline suggesting no effect of these deletions on basal locomotion (Table 4, item a). However, a significant dose by genotype interaction was found following oxycodone $(p<0.001$, Table 4 , item $b)$. (1) Dose. When compared with the saline group of the same genotype, the $1 \mathrm{mg} / \mathrm{kg}$ dose of oxycodone had no effect, but 3 and $10 \mathrm{mg} / \mathrm{kg}$ of oxycodone induced hyperlocomotion in control flMORs $(p=0.007$ and $p=0.0002$, respectively), A2afIMORs ( $p<0.0001$ for both doses), and following $10 \mathrm{mg} /$ $\mathrm{kg}$ in D2fIMORs $(p<0.0001)$ and ChATfIMORs $(p=0.0013)$. However, there was no effect of oxycodone in the D1flMORs (Table 4, item b). (2) Genotype. D1flMORs showed a decreased response compared with fIMORs at $10 \mathrm{mg} / \mathrm{kg}$ $(p=0.04)$ whereas A2aflMORs showed a greater locomotor response than flMORs $(p<0.0001$ for both doses). Neither ChATfIMORs nor D2fIMORs differed from fIMORS (Table 4, item c).

\section{Morphine}

Our first experiments examined the dose-dependent locomotor effects of morphine using 0 (saline), 3,10 , and 
Ai

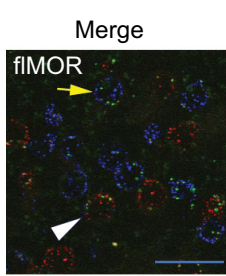

drd1
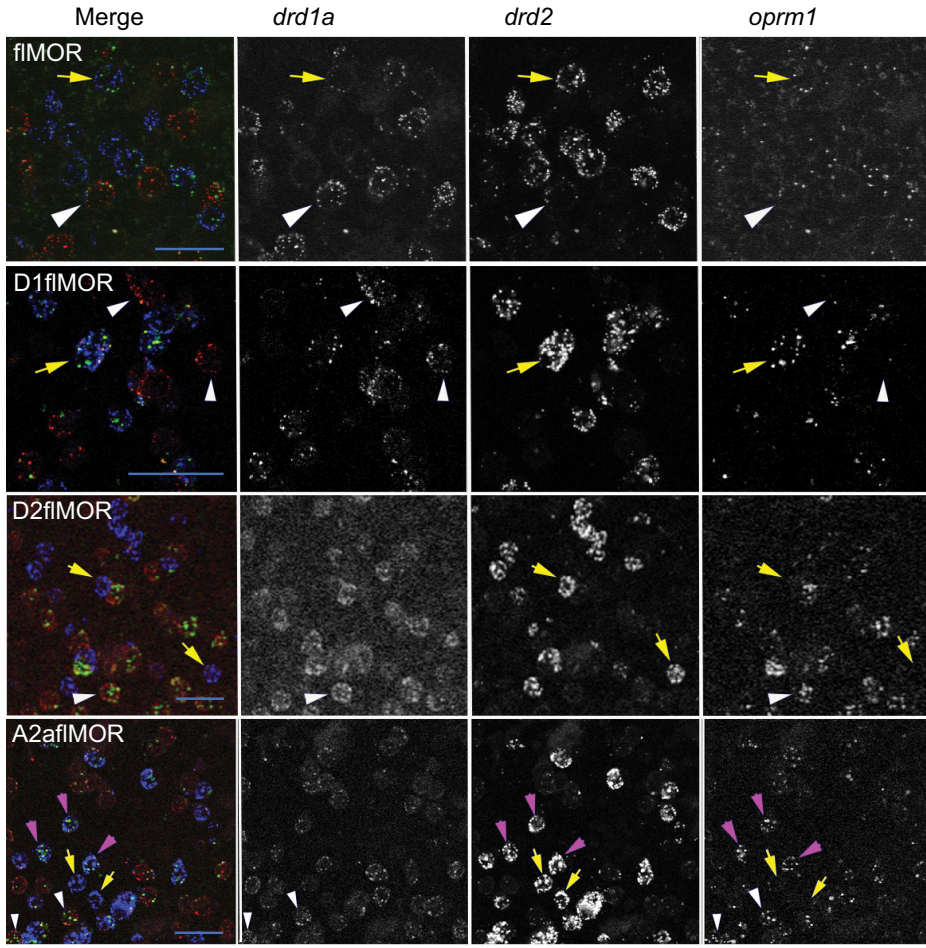

Aii

\begin{abstract}
Merge
\end{abstract}
drd1a
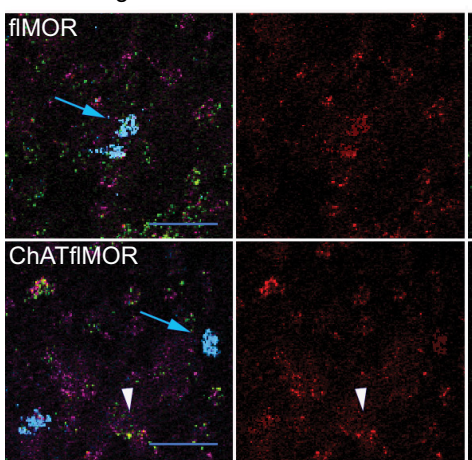

Ci

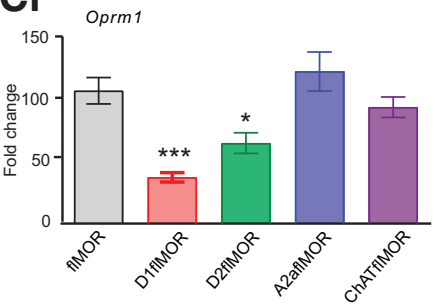

ChAT

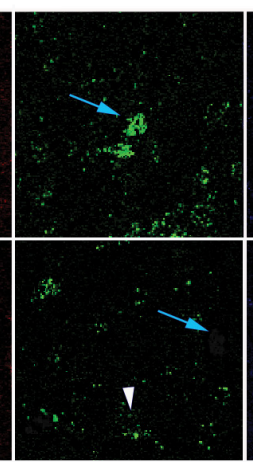

Cii

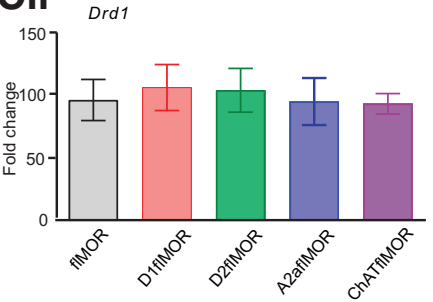

$\mathrm{Bi}$

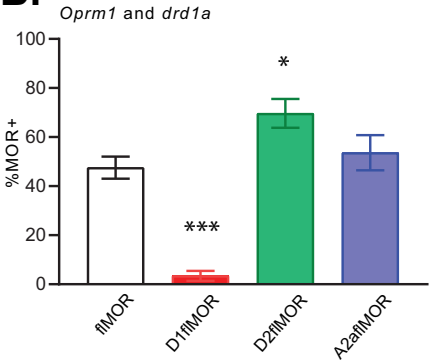

Bii

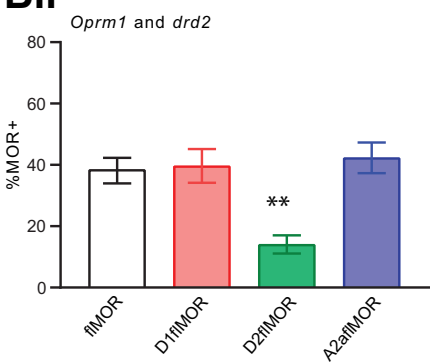

Biii

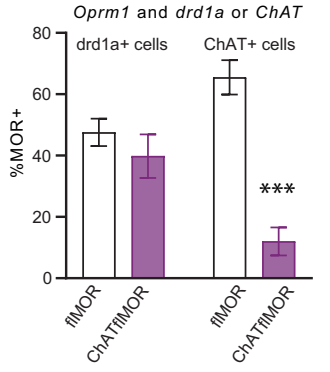

Figure 1. Validation of the selectivity and extent of MOR knock-down in striatal subpopulations. Ai, Representative RNA in situ hybridization images for MOR (oprm1 in white), dopamine receptor 1 (D1 or drd1a in red) and dopamine receptor 2 (D2 or drd2 in green), are shown from the dorsolateral striatum of control, fIMOR, and D1fIMOR, D2fIMOR, and A2afIMOR mouse lines. White arrows without a tail demonstrate D1-expressing cells and yellow arrows with a tail show D2-expressing cells. The cells marked by pink arrows in the A2afIMOR images show cells that are oprm1 and drd2 positive. Aii, Representative RNA in situ hybridization images of the dorsolateral striatum showing oprm1 (white), ChAT (green), and D1 (red) labeling in fIMOR and ChATfIMOR lines. Arrows highlight ChAT+ cells. Scale bar $=20 \mu \mathrm{M}(\boldsymbol{A} \boldsymbol{i}, \boldsymbol{A} \boldsymbol{A i}) . \boldsymbol{B}$, Oprm1 expression were quantified and presented as the \% colocalization for each genotype of MOR with D1+ cells in $\mathbf{B i}$, MOR with D2+ cells in Bii, and MOR with ChAT+ or D1+ cells in Bii; $* p<0.05, * * p<$ 0.01 , and $* * * p<0.001$ versus fIMORs of the same experiment. $\mathbf{C}$, qPCR for oprm1, drd1, and drd2 cDNA levels in striatal tissue shows reduced oprm1expression in D1fIMORs $(* * * p<0.001)$ and D2fIMORs $(* p<0.05)$ compared with fIMORs. There was no effect of these MOR deletions on drd1 (Cii) or drd2 (Ciii) expression. Refer to Table 2 for statistical analyses. All data are shown as mean \pm SEM, and the individual datapoints are shown in Extended Data Figure 1-1, for which this legend also applies. 
Table 2: Statistical analyses of MOR knock-down in D1-, D2-, A2a-, and ChAT-fIMOR lines by RNA ISH and qPCR (Fig. 1)

\begin{tabular}{|c|c|c|c|c|c|c|c|c|c|c|}
\hline Item & Figure & Experiment & Statistical test & Effect or interaction & Main effect & flMOR & D1fIMOR & D2fIMOR & A2afIMOR & ChATfIMOR \\
\hline $\mathbf{a}$ & $1 B i$ & $\begin{array}{l}\text { RNA in situ } \\
\text { hybridization }\end{array}$ & One-way ANOVA & $\begin{array}{l}\text { Genotype, oprm } 1 \text { and } \\
\text { drd1 probes }\end{array}$ & $\begin{array}{r}F_{(3,16)}=29.14 \\
p<0.0001\end{array}$ & $\begin{array}{l}\text { Reference genotype } \\
\quad n=5\end{array}$ & $\begin{array}{c}p<0.0001 \\
n=5\end{array}$ & $\begin{array}{r}p=0.02 \\
n=5\end{array}$ & $\begin{array}{l}\text { N.S. } \\
\quad n=5\end{array}$ & N.A. \\
\hline b & $1 B i i$ & $\begin{array}{l}\text { RNA in situ } \\
\text { hybridization }\end{array}$ & One-way ANOVA & $\begin{array}{l}\text { Genotype, oprm } 1 \text { and } \\
\text { drd2 probes }\end{array}$ & $\begin{array}{l}F_{(3,15)}=8.76 \\
p=0.0013\end{array}$ & $\begin{array}{l}\text { Reference genotype } \\
\quad n=5\end{array}$ & $\begin{array}{c}p<0.99 \\
n=5\end{array}$ & $\begin{array}{c}p=0.004 \\
n=5\end{array}$ & $\begin{array}{l}\text { N.S. } \\
\qquad n=4\end{array}$ & N.A. \\
\hline c & $1 B i i$ & $\begin{array}{l}\text { RNA in situ } \\
\text { hybridization }\end{array}$ & One-way ANOVA & $\begin{array}{c}\text { Genotype, oprm1, drd1 } \\
\text { and ChAT probes }\end{array}$ & $\begin{array}{c}F_{(3,13)}=15.95 \\
p=0.0001\end{array}$ & $\begin{array}{l}\text { Reference genotype } \\
\quad n=4\end{array}$ & N.A. & N.A. & N.A. & $\begin{array}{l}\text { ChAT+ vs -; } \\
\begin{array}{l}p<0.0001, \\
n=4 \\
\text { D1+ vs -; NS } \\
n=5 \text { and } 4\end{array}\end{array}$ \\
\hline d & $1 \mathrm{Ci}$ & qPCR & One-way ANOVA & Genotype, oprm1 probe & $\begin{array}{r}F_{(4,35)}=8.59 \\
p<0.0001\end{array}$ & $\begin{array}{l}\text { Reference genotype } \\
\quad n=13\end{array}$ & $p=0.0002, n=6$ & $\begin{array}{c}p=0.015 \\
n=8\end{array}$ & N.S., $n=6$ & N.S., $n=7$ \\
\hline e & $1 C i i$ & qPCR & One-way ANOVA & Genotype, drd1 probe & $\begin{array}{l}F_{(6,26)}=0.339 \\
\text { N.S. }\end{array}$ & $\begin{array}{l}\text { Reference genotype } \\
\quad n=6\end{array}$ & N.S., $n=6$ & N.S., $n=5$ & N.S., $n=5$ & N.S., $n=6$ \\
\hline f & 1Ciii & qPCR & One-way ANOVA & Genotype, $d r d 2$ probe & $\begin{array}{l}F_{(5,29)}=0.925 \\
\quad \text { N.S. }\end{array}$ & $\begin{array}{l}\text { Reference genotype } \\
\qquad n=10\end{array}$ & N.S., $n=5$ & N.S., $n=7$ & N.S., $n=5$ & N.S., $n=6$ \\
\hline
\end{tabular}

The RNA probes used were; oprm1 (MOR), drd1 (D1 receptor), drd2 (D2 receptor), and ChAT (cholineacetyltransferase) in fIMOR, D1-, D2-, A2a-, and ChATfIMOR lines. The qPCR probes used were oprm1 (MOR), drd1 (D1 receptor), drd2 (D2 receptor) in fIMOR, D1-, D2-, A2a-, and ChAT-fIMOR lines. RNA ISH: RNA in situ hybridization, N.S.: not significant, N.A: not applicable

$15 \mathrm{mg} / \mathrm{kg}$ (subcutaneously; Fig. 3B). We observed a dose $\times$ genotype interaction ( $p<0.001$, Table 4 , item $d$ ) as follows. (1) Dose. When compared with the group receiving saline of the same genotype, we found that $15 \mathrm{mg} / \mathrm{kg}$ morphine, but not any lower doses, induced hyperlocomotion in fIMORs $(p=0.003)$ and D2fIMORs $(p<0.0001)$. D1fIMORs and ChATfIMORs showed no response at any dose (Table 4, item d) whereas A2aflMORs showed hyperlocomotion after both 10 and $15 \mathrm{mg} / \mathrm{kg}(p<0.0001$ for both doses), but not 3 $\mathrm{mg} / \mathrm{kg}$. (2) Genotype. Between genotype analysis (Table 4, item e) showed a similar effect of genotype following morphine as oxycodone treatment in that, when compared with flMORs, A2aflMORs showed an enhanced response at the higher doses used, $10(p=0.0001)$ and $15(p=0.004) \mathrm{mg} / \mathrm{kg}$, whereas D1fIMORs showed a reduced response at $15 \mathrm{mg} / \mathrm{kg}$ $(p=0.004)$, but not $10 \mathrm{mg} / \mathrm{kg}$. Both D2- and ChAT-fIMORs were not different from flMORs.

\section{Cocaine}

To assess whether the changes in opioid-induced locomotor responses were generalizable to other drug classes, we determined the effect of genotype on cocaine-induced locomotion ( $15 \mathrm{mg} / \mathrm{kg}$, s.c.; Fig. $3 C$ ). We found cocaine-induced locomotion in all genotypes (Fig. $3 C, p \leq 0.001$; Table 4, item f) but this effect was enhanced in ChATfIMORs $(p<0.0005$, Table 4 , item $g)$.

\section{Locomotor sensitization}

Repeated opioid exposure is well known to induce a sensitization of the initial hyperlocomotor response (Tao et al., 2017). This occurs concurrently with an increase in the incentive motivational properties of a drug and has been considered as a window into this property of drugseeking behavior (Robinson and Berridge, 1993). To assess the role of each of these MOR populations in this phenomenon, we examined sensitization to oxycodone (10 mg/kg, s.c.), morphine (15 mg/kg, s.c.), and saline, over three consecutive days of drug exposure in all genotypes. The data were analyzed by two-way ANOVA to assess the effect of day and drug on the first and last days of the test. The fIMORs showed a genotype $\times$ day interaction as both oxycodone $(p=0.002)$ and morphine $(p=$ 0.02 ), but not saline, induced sensitization (Fig. 3D; Table 4 , item h). The D1flMORs showed no sensitization effect following oxycodone or morphine and this response was not different from saline (Fig. 3E; Table 4, item i). The D2fIMORs were similar to fIMORs as they sensitized to both oxycodone $(p<0.0001)$ and morphine $(p<0.0001)$ but not saline (Fig. 3F; Table 4, item j). The A2aflMORs sensitized to oxycodone $(p<0.0001)$ but not to morphine or saline. (Fig. 3G; Table 4, item k). The ChATfIMORs similarly sensitized to oxycodone $(p<0.0001)$, but not morphine or saline (Fig. $3 \mathrm{H}$; Table 4, item I).

\section{Intrasession locomotor activity}

We then defined the locomotion profile induced by each drug with each session using linear mixed model analysis to assess the effect of time and genotype. This was done using 5-min timebins on day 1 and day 3 of $10 \mathrm{mg} / \mathrm{kg}$ oxycodone or $15 \mathrm{mg} / \mathrm{kg}$ morphine. (1) There was a genotype $\times$ time interaction on day 1 of oxycodone (Fig. 3l, $p<0.0001$; Table 4, item m). The D2flMORs (Table 4, item $n, p=0.02$ ) and A2aflMORs $(p<0.0001)$, but not fIMORs, D1- or ChAT-fIMORs showed a change in locomotor activity within the session. (2) We did not find a timebin $\times$ genotype interaction (Table 4 , item o) on day 3 of oxycodone. However, the D1flMORs showed decreased activity over time (Fig. 3J, $p<0.001$; Table 4, item p), but no other change in activity over time was observed in other lines. (3) There was a genotype $\times$ time interaction on day 1 of morphine (Fig. 3K, $p<0.0001$; Table 4, item q), with D1fIMORs showing a different locomotor profile than fIMORs $(p=0.002)$. Further post hoc analyses showed that fIMORs (Table 4, item $r, p<0.0001)$, D2fIMORs ( $p<0.0001)$, A2afIMORs $(p<0.0001)$, and ChATfIMORs $(p=0.002)$, but not D1fIMORs, increased their locomotor activity during the session. (4) We also observed a significant genotype $\times$ time interaction on day 3 (Fig. $3 L, p<0.0001$; Table 4, item s), with both D1fIMOR $(p<0.0001)$ and ChATfIMOR $(p<0.001)$ showing less activity during the session than 
A
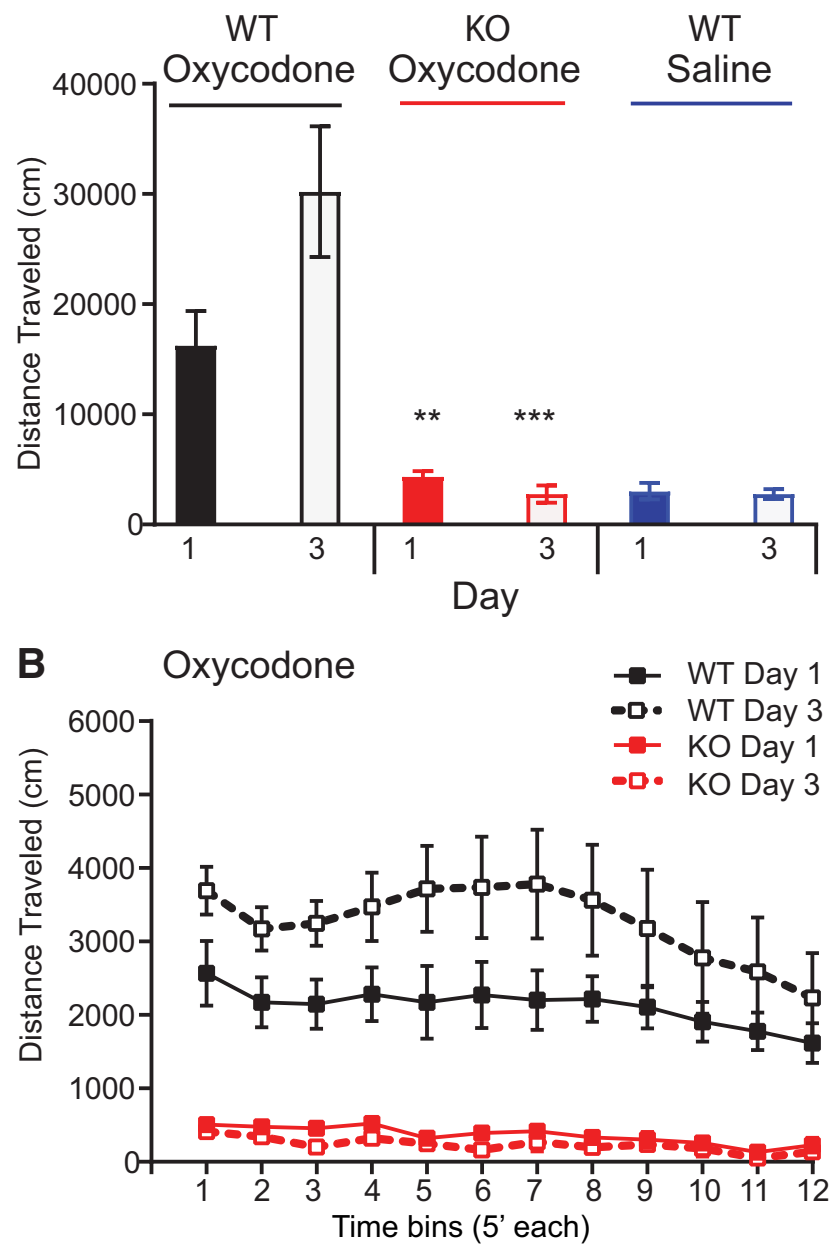

Figure 2. Oxycodone-induced locomotion is absent in constitutive MOR KOs. A, Oxycodone (10 mg/kg, s.c.) induces hyperlocomotion in WT mice (WT) that is absent in mice lacking all MORs (KO) on the first $(* * p<0.01$ vs WT) and third (***p $<0.001$ vs WT) days of three consecutive days of oxycodone. There was no difference between the effect of oxycodone in $\mathrm{KO}$ mice with that of saline in WT mice $(p=0.92)$. $B$, The intrasession data (shown here in 5 -min timebins for the 60-min test) further show the lack of effect of oxycodone in KO mice on days 1 and $3(p<0.0001$ vs WT at all timepoints for both days). WTs demonstrated a sensitization of this locomotor response $(p<0.01)$ from day 1 to day 3 that was absent in KOs. Refer to Table 3 for statistical analyses. All data are shown as mean \pm SEM, and the individual datapoints are shown in Extended Data Figure 2-1 for which this legend also applies. the fIMORs. Further post hoc analyses showed that flMORs (Table 4, item t, $p<0.0001)$, D2flMORs $(p<$ $0.0001)$, A2afIMORs $(p<0.0001)$, and ChATfIMORs $(p=$ 0.03 ), but not D1fIMORs, increased their locomotor activity during this session.

\section{Selective MOR deletions define specific roles of A2a and ChAT MOR populations in opioid IVSA}

Although opioid-induced locomotion and sensitization of this response have been used as an index of reward behaviors (Robinson and Berridge, 1993; Stewart and Badiani, 1993), IVSA is considered as a more direct measure of reward seeking and addiction (Everitt et al., 2018). We therefore examined whether deleting MORs from these neurons altered opioid IVSA through an indwelling jugular catheter under a short-access FR1 schedule. Each of the phases of the IVSA protocol (remifentanil acquisition, oxycodone maintenance and extinction) were analyzed separately and results presented for each of the following four parameters; active and inactive lever presses, reinforcers earned and lever choice as shown by the percent of active lever/total lever presses made.

\section{Remifentanil acquisition}

Remifentanil, a fast-acting opioid, was used to establish the association of an active lever press with an opioid infusion and associated cues. During this short acquisition phase, we did not find a genotype $x$ day interaction or any main effect of genotype on any of the four parameters measured; (Fig. $4 A-D$, respectively). However, we found a main effect of day on active lever presses made $(p<$ $0.0001, \chi^{2}=21.017$; Table 5, item a), reinforcers earned $\left(p<0.0001, \chi^{2}=19.132\right.$; Table 5, item b), and percentage active lever presses $\left(p<0.01, \chi^{2}=9.730\right.$; Table 5 , item c), but not inactive lever presses, showing that all lines acquired this self-administration behavior but there was no effect of genotype.

\section{Oxycodone maintenance}

The mice were then transitioned to oxycodone selfadministration for $9 \mathrm{~d}$. Compared with those on saline, mice receiving oxycodone made more active lever presses (Fig. 4E, $p<0.001$; Table 5 , item d), earned more reinforcers (Fig. 3G, $p<0.0001$; Table 5, item e), and had a higher percentage active lever presses (Fig. $4 H$, $p<0.0001$; Table 5 , item f). There was no difference in the inactive lever presses made between the saline and

Table 3: Statistical analyses of the hyperlocomotor effects of oxycodone in MOR KO mice (Fig. 2)

\begin{tabular}{|c|c|c|c|c|c|c|c|}
\hline Item & Figure & Experiment & Statistical test & Effect or Interaction & Main effect & WT & $\mu \mathrm{KO}$ \\
\hline a & $2 A$ & Oxycodone; total locomotion on day 1 & Two-way ANOVA & Genotype, $\mu$ KO vs WT & $F_{(2,26)}=10.78, p<0.0004$ & $n=7$, day 1 & $\begin{array}{c}p<0.01 \text { vs WT oxycodone, } n=9 \\
p=0.92 \text { vs WT saline, } n=8\end{array}$ \\
\hline b & $2 A$ & Oxycodone sensitization; total locomotion & Two-way ANOVA & Genotype, $\mu$ KO vs WT & $F_{(2,26)}=5.6, p=0.0095$ & $\begin{array}{l}p<0.001 \text { day } 1 \text { vs } \\
\quad 3 n=7\end{array}$ & $p=0.97$ day 1 vs $3, n=9$ \\
\hline c & $2 B$ & $\begin{array}{l}\text { Oxycodone sensitization; intrasession } \\
\text { analysis }\end{array}$ & Two-way ANOVA & Genotype, $\mu$ KO vs WT & $F_{(33,308)}=1.67, p=0.015$ & $\begin{array}{l}p<0.01, \text { day } 1 \text { vs } \\
\quad 3, n=7\end{array}$ & $\begin{array}{l}\mu \mathrm{KO} \text { vs WT days } 1 \text { and } 3 \\
\quad p<0.001, n=9\end{array}$ \\
\hline
\end{tabular}

N.S.: not significant, N.A.: not applicable. 

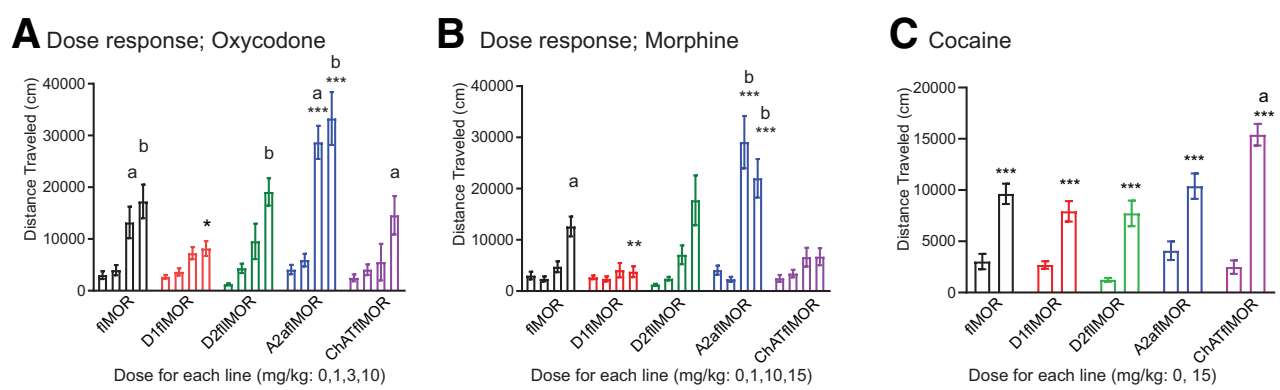

D Sensitization; fIMOR

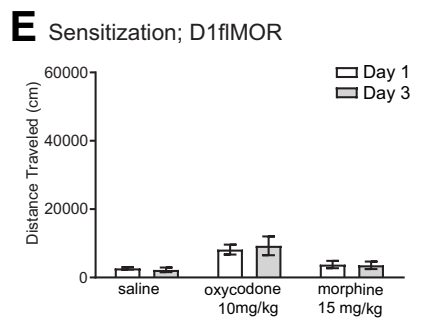

F Sensitization; D2fIMOR

G Sensitization; A2aflMOR
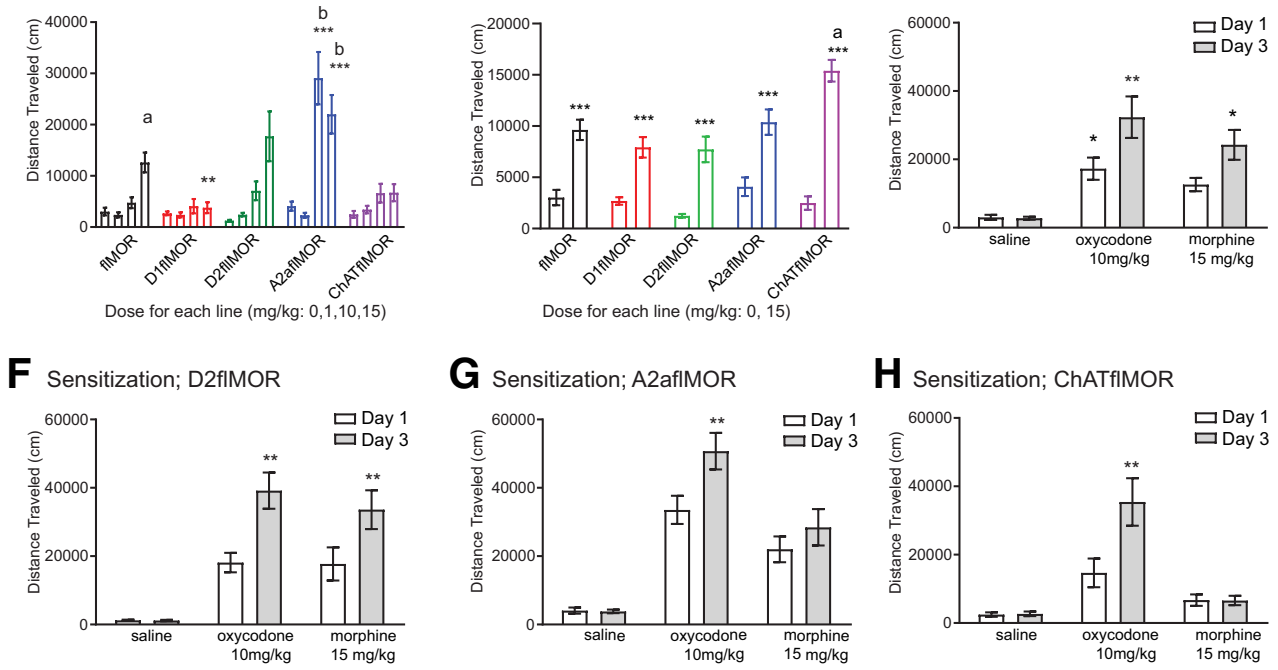

H Sensitization; ChATfIMOR
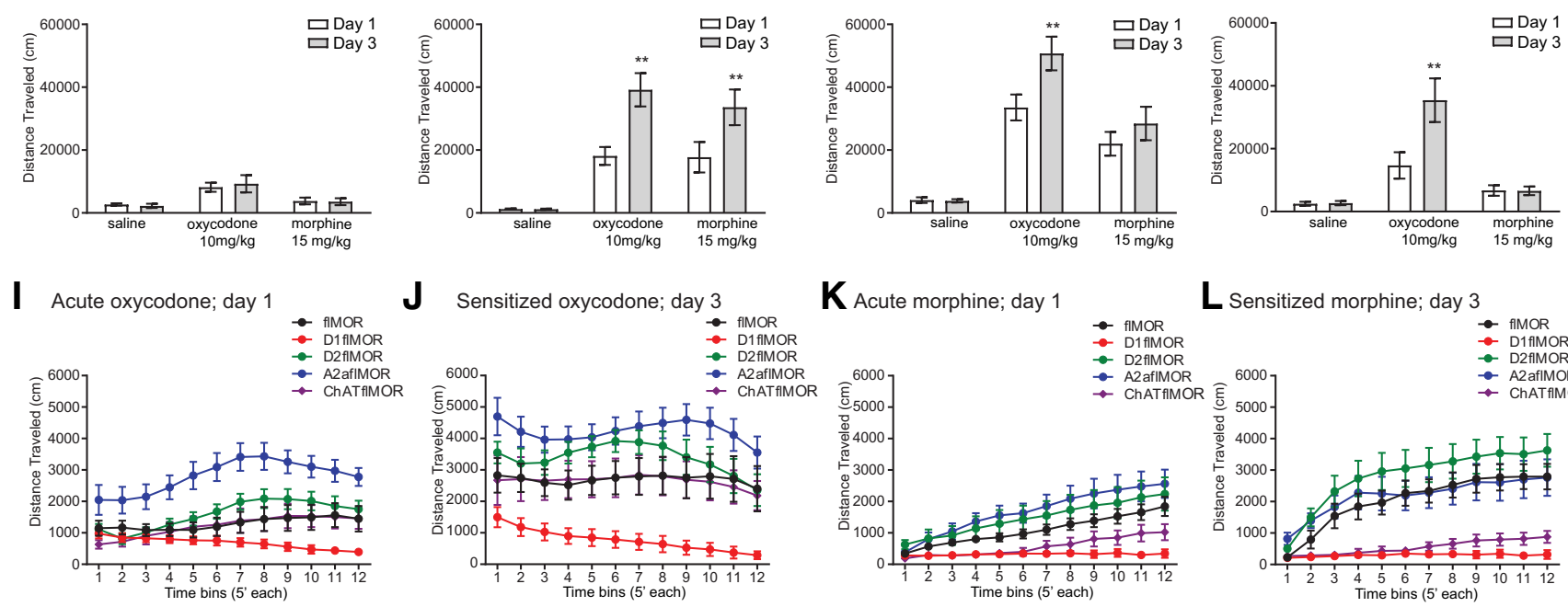

L Sensitized morphine; day 3

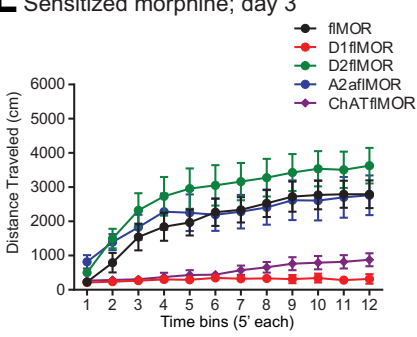

Figure 3. Selective MOR deletions define specific roles of D1 and A2a MOR populations in opioid-induced locomotion. $\boldsymbol{A}$, Oxycodone $(0,1,3,10 \mathrm{mg} / \mathrm{kg})$ induced a dose-dependent increase in locomotion in flMORs, D2fIMORs, A2aflMORs, and ChATfIMORs, but not D1fIMORs (a: $p<0.01$ vs 0 , b: $p<0.001$ vs 0). In comparison with the control genotype, fIMORs, D1fIMORs showed a reduced effect of oxycodone at $10 \mathrm{mg} / \mathrm{kg}(* p<0.05 \mathrm{vs}$ fIMOR of the same dose), whereas A2aflMORs showed an enhanced effect of oxycodone at 3 and $10 \mathrm{mg} / \mathrm{kg}(* * * p<0.001 \mathrm{vs}$ fIMOR of the same dose). There was no effect of genotype following the vehicle (0) injection showing no effect of any of these deletions on basal locomotor activity. $\boldsymbol{B}$, Morphine $(0,1,10,15 \mathrm{mg} / \mathrm{kg})$ also induced a dose-dependent increase in locomotor activity in fIMORs, D2fIMORs, and A2afIMORs but not in D1fIMORs or ChATfIMORs (a: $p<0.01$ vs 0 , b: $p<0.001$ vs 0 ). Compared with control fIMORs, this effect was enhanced in A2afIMORs $(* * p<0.001$ and $* * * p<0.0001$ vs flMOR of the same dose). C, Cocaine $(0,15 \mathrm{mg} / \mathrm{kg})$ induced hyperlocomotion in all lines when compared with saline $(0 ; * * * p \leq 0.001)$, an effect that was enhanced in ChATfIMORs (a: $p<0.001$ vs fIMORs). $\boldsymbol{D}-\boldsymbol{H}$, Sensitization. After three consecutive days of repeated opioid injections, flMORs $(\boldsymbol{D})$ and D2flMORs $(\boldsymbol{F})$ showed an enhanced, or sensitized, response to both oxycodone and morphine. D1fIMORs $(\boldsymbol{E})$ did not show this enhanced effect to either opioid whereas A2aflMORs $(\boldsymbol{G})$ and ChATfIMORs $(\boldsymbol{H})$ sensitized to oxycodone but not morphine $(* p<0.05$ and $* * p<0.01$, respectively, vs day 1$)$. I $\boldsymbol{L}$, Intrasession locomotor analysis. This analysis assessed the locomotor response to oxycodone or morphine during each 60-min session on day 1 and day 3. I, A single injection of oxycodone $(10 \mathrm{mg} / \mathrm{kg})$ on day 1 increased locomotor activity in D2flMORs $(p<0.05)$ and A2afIMORs $(p<0.0001)$, whereas fIMORs, D1fIMORs, and ChATfIMORs showed no change in activity during the session. J, After $3 \mathrm{~d}$ of repeated oxycodone administration, the locomotor activity of D1fIMORs $(p<0.001)$ declined through the session and all other genotypes showed no change across time. $\boldsymbol{K}$, A single injection of morphine $(15 \mathrm{mg} / \mathrm{kg})$ on day 1 resulted in a within-session increase in locomotor activity in fIMORs $(p<0.0001)$, D2fIMORs $(p<0.0001)$, A2afIMORs $(p<0.0001)$, and ChATfIMORs $(p<0.01)$, but not D1fIMORs. $L$, After $3 \mathrm{~d}$ of repeated morphine administration, a similar pattern emerged as on day 1 with fIMORs $(p<0.0001)$, D2fIMORs $(p<0.0001)$, A2afIMORs $(p<0.0001)$, and ChATfIMORs $(p<0.05)$, but not D1fIMORs, showing a within session increase in locomotor activity. Refer to Table 4 for statistical analyses. All data are shown as mean \pm SEM, and individual datapoints are shown in Extended Data Figure 3-1 for which this legend also applies.

oxycodone groups (Fig. 4F). There was no effect of genotype on any parameter.

\section{Extinction}

Extinction has been shown to increase drug-seeking behavior following oxycodone self-administration (Hakimian et al., 2019). We similarly found that, when compared with saline, all genotypes showed a treatment $x$ day interaction in the number of active lever presses made $(p<0.01$; Fig. $4 E$;
Table 5, item g) and reinforcers earned $(p<0.01$; Fig. 4G; Table 5, item h), but not inactive lever presses (Fig. $4 F$ ) or percentage active lever presses (Fig. 4D) between the last day of oxycodone maintenance and the first day of extinction. Post hoc analyses showed an effect of oxycodone in that mice receiving oxycodone made more active lever presses $(p<0.0001$; Table 5 , item i), inactive lever presses $(p<0.0001$, Table 5, item j) and earned more reinforcers $(p<0.0001$; Table 5 , item $k)$ on the first day of extinction 
Table 4: Statistical analyses of the hyperlocomotor effects of oxycodone, morphine, and cocaine in all lines (Fig. 3)

\begin{tabular}{|c|c|c|c|c|c|c|c|c|c|c|}
\hline Item & Figure & Experiment & $\begin{array}{l}\text { Statistical } \\
\text { test }\end{array}$ & $\begin{array}{l}\text { Effect or } \\
\text { Interaction }\end{array}$ & Main effect & fIMOR & D1fIMOR & D2fIMOR & A2aflMOR & ChATfIMOR \\
\hline a & $3 A$ & $\begin{array}{l}\text { Oxycodone dose re- } \\
\text { sponse: dose re- } \\
\text { sponse }(0 \mathrm{mg} / \mathrm{kg} \text { or } \\
\text { saline) }\end{array}$ & $\begin{array}{l}\text { One-way } \\
\text { ANOVA }\end{array}$ & Genotype & $\begin{array}{c}F_{(4,36)}=2.54 \\
p=0.056\end{array}$ & $\begin{array}{l}\text { Reference geno- } \\
\quad \text { type } n=8\end{array}$ & N.S. $n=8$ & N.S. $n=8$ & N.S. $n=8$ & N.S. $n=9$ \\
\hline b & $3 A$ & $\begin{array}{l}\text { Oxycodone dose re- } \\
\text { sponse: dose effect }\end{array}$ & $\begin{array}{l}\text { Two-way } \\
\text { ANOVA }\end{array}$ & $\begin{array}{l}\text { Genotype } \times \\
\text { dose }\end{array}$ & $\begin{array}{c}F_{(12,145)}=3.76 \\
p<0.001\end{array}$ & $\begin{array}{l}3 \mathrm{mg} ; p=0.007 \text { vs } \\
\begin{array}{l}0,10 \mathrm{mg} ; \\
p=0.0002 \text { vs } 0 \\
n=8-12\end{array}\end{array}$ & N.S. $n=8-11$ & $\begin{array}{c}3 \mathrm{mg} ; p=0.56 \text { vs } \\
0,10 \mathrm{mg} ; p< \\
0.0001 \text { vs } 0, \\
n=8\end{array}$ & $\begin{array}{c}3 \mathrm{mg} ; p<0.0001 \text { vs } \\
\quad 0,10 \mathrm{mg} ; p< \\
0.0001 \text { vs } 0 \\
n=6-9\end{array}$ & $\begin{array}{c}3 \mathrm{mg} ; p=0.80 \text { vs } 0 \\
10 \mathrm{mg} ; p=0.0013 \\
\text { vs } 0 n=5-9\end{array}$ \\
\hline c & $3 A$ & $\begin{array}{l}\text { Oxycodone dose re- } \\
\text { sponse: genotype } \\
\text { effect }\end{array}$ & $\begin{array}{l}\text { Two-way } \\
\text { ANOVA }\end{array}$ & $\begin{array}{l}\text { Genotype } \times \\
\text { dose }\end{array}$ & $\begin{array}{c}F_{(12,145)}=3.76 \\
p<0.001\end{array}$ & $\begin{array}{l}\text { Reference } \\
\text { genotype }\end{array}$ & $10 \mathrm{mg}: p=0.04$ & N.S. & $\begin{array}{c}3 \mathrm{mg} ; p=0.0001,10 \\
\mathrm{mg} ; p<0.0001\end{array}$ & N.S. \\
\hline d & $3 B$ & $\begin{array}{l}\text { Morphine dose re- } \\
\text { sponse: dose effect }\end{array}$ & $\begin{array}{l}\text { Two-way } \\
\text { ANOVA }\end{array}$ & $\begin{array}{l}\text { Genotype } \times \\
\text { dose }\end{array}$ & $\begin{array}{c}F_{(12,148)}=5.7 \\
p<0.001\end{array}$ & $\begin{array}{c}10 \mathrm{mg} ; \mathrm{N} . \mathrm{S} .15 \\
\mathrm{mg} ; p=0.003 \\
\text { vs } 0 n=8-11\end{array}$ & N.S. $n=8-11$ & $\begin{array}{l}10 \text { mg; N.S. } 15 \\
\text { mg; } p<0.0001 \\
n=8-11\end{array}$ & $\begin{array}{c}10 \mathrm{mg} ; p<0.0001 \\
15 \mathrm{mg} ; p< \\
0.0001 n=5-9\end{array}$ & N.S. $n=6-9$ \\
\hline e & $3 B$ & $\begin{array}{l}\text { Morphine dose re- } \\
\text { sponse: genotype } \\
\text { effect }\end{array}$ & $\begin{array}{l}\text { Two-way } \\
\text { ANOVA }\end{array}$ & $\begin{array}{l}\text { Genotype } \times \\
\text { dose }\end{array}$ & $\begin{array}{c}F_{(12,148)}=5.7 \\
p<0.001\end{array}$ & $\begin{array}{l}\text { Reference } \\
\text { genotype }\end{array}$ & $15 \mathrm{mg}: p=0.004$ & N.S. & $\begin{array}{r}10 \mathrm{mg} ; p=0.0001 \\
15 \mathrm{mg} ; p=0.004\end{array}$ & N.S. \\
\hline f & $3 C$ & Cocaine; dose effect & $\begin{array}{r}\text { Two-way } \\
\text { ANOVA }\end{array}$ & $\begin{array}{r}\text { Treatment } \times \\
\text { genotype }\end{array}$ & $\begin{array}{l}F_{(4,83)}=3.77 \\
p=0.0073\end{array}$ & $\begin{array}{l}p<0.0001 \\
\quad \text { Cocaine, } n= \\
21 ; \text { saline } n=9\end{array}$ & $\begin{array}{c}p=0.0018 \text { Cocaine }, \\
\quad n=9 ; \text { saline } n= \\
14\end{array}$ & $\begin{array}{l}p=0.0010 \\
\quad \text { Cocaine, } n=9 \\
\quad \text { saline } n=7\end{array}$ & $\begin{array}{r}p=0.0005 \text { Cocaine } \\
n=9 ; \text { saline } n=9\end{array}$ & $\begin{array}{c}p<0.0001 \text { Cocaine } \\
\quad \begin{array}{l}n=10 ; \text { saline } \\
n=7\end{array}\end{array}$ \\
\hline g & $3 C$ & $\begin{array}{l}\text { Cocaine; genotype } \\
\text { effect }\end{array}$ & $\begin{array}{r}\text { Two-way } \\
\text { ANOVA }\end{array}$ & $\begin{array}{r}\text { Treatment } \times \\
\text { genotype }\end{array}$ & $\begin{array}{l}F_{(4,83)}=3.77 \\
p=0.0073\end{array}$ & $\begin{array}{l}\text { Reference } \\
\text { genotype }\end{array}$ & $\begin{array}{l}\text { N.S. for saline and } \\
\text { cocaine }\end{array}$ & $\begin{array}{l}\text { N.S. for saline and } \\
\text { cocaine }\end{array}$ & $\begin{array}{l}\text { N.S. for saline and } \\
\text { cocaine }\end{array}$ & $\begin{array}{l}\text { Saline; N.S. Cocaine; } \\
\quad p<0.0005\end{array}$ \\
\hline h & $3 D$ & $\begin{array}{l}\text { Locomotor sensitiza- } \\
\text { tion; flMORs }\end{array}$ & $\begin{array}{l}\text { Two-way } \\
\text { ANOVA }\end{array}$ & $\begin{array}{l}\text { Genotype } \times \\
\text { day }\end{array}$ & $\begin{array}{r}F_{(2,27)}=3.9 \\
p=0.049\end{array}$ & $\begin{array}{l}\text { Oxycodone; } p= \\
\begin{array}{l}0.002 \text { mor- } \\
\text { phine; } p= \\
0.018 n=11 \text { for } \\
\text { both, saline } \\
n=8\end{array}\end{array}$ & & & & \\
\hline i & $3 E$ & $\begin{array}{l}\text { Locomotor sensitiza- } \\
\text { tion; D1fIMORs }\end{array}$ & $\begin{array}{l}\text { Two-way } \\
\text { ANOVA }\end{array}$ & $\begin{array}{l}\text { Genotype } \times \\
\text { day }\end{array}$ & $\begin{array}{r}F_{(2,25)}=0.6 \\
p=0.56\end{array}$ & & $\begin{array}{c}\text { N.S. oxycodone } n= \\
8, \text { morphine } n= \\
11, \text { saline } n=8\end{array}$ & & & \\
\hline j & $3 F$ & $\begin{array}{l}\text { Locomotor sensitiza- } \\
\text { tion; D2fIMORs }\end{array}$ & $\begin{array}{l}\text { Two-way } \\
\text { ANOVA }\end{array}$ & $\begin{array}{l}\text { Genotype } \times \\
\text { day }\end{array}$ & $\begin{array}{c}F_{(2,23)}=12.24 \\
p=0.0002\end{array}$ & & & $\begin{array}{c}\text { Oxycodone; } p< \\
0.0001, n=7 \\
\text { morphine; } p< \\
0.0001 n=11, \\
\text { saline } n=8\end{array}$ & & \\
\hline k & $3 G$ & $\begin{array}{l}\text { Locomotor sensitiza- } \\
\text { tion; A2aflMORs }\end{array}$ & $\begin{array}{l}\text { Two-way } \\
\text { ANOVA }\end{array}$ & $\begin{array}{l}\text { Genotype } \times \\
\text { day }\end{array}$ & $\begin{array}{r}F_{(2,25)}=8.23 \\
p=0.0018\end{array}$ & & & & $\begin{array}{l}\text { Oxycodone; } p< \\
0.0001 n=11, \\
\text { morphine; } p= \\
0.15, n=9, \text { saline } \\
n=8\end{array}$ & \\
\hline I & $3 H$ & $\begin{array}{l}\text { Locomotor sensitiza- } \\
\text { tion; ChATfIMORs }\end{array}$ & $\begin{array}{l}\text { Two-way } \\
\text { ANOVA }\end{array}$ & $\begin{array}{l}\text { Genotype } \times \\
\text { day }\end{array}$ & $\begin{array}{c}F_{(2,23)}=11.53 \\
p=0.0003\end{array}$ & & & & & $\begin{array}{l}\text { Oxycodone; } p< \\
\begin{array}{l}0.0001, n=8, \\
\text { morphine; N.S., } \\
n=9, \text { saline; } n=9\end{array}\end{array}$ \\
\hline m & 31 & $\begin{array}{l}\text { Oxycodone intrases- } \\
\text { sion analysis: Day } 1\end{array}$ & LMM & $\begin{array}{c}\text { Genotype } \times \\
\text { timebin }\end{array}$ & $\begin{array}{r}\chi^{2}=11.882 \\
p=0.018\end{array}$ & N.A. & N.S., $n=9$ & N.S., $n=8$ & N.S., $n=11$ & N.S., $n=9$ \\
\hline $\mathbf{n}$ & $3 \mathrm{~J}$ & $\begin{array}{l}\text { Oxycodone intrases- } \\
\text { sion analysis: day } 1\end{array}$ & LMM & Timebin & $\begin{aligned} \chi^{2} & =31.215 \\
p & <0.0001\end{aligned}$ & N.S., $n=11$ & $p=0.076, n=9$ & $p=0.016, n=8$ & $p<0.0001, n=11$ & N.S., $n=9$ \\
\hline o & $3 K$ & $\begin{array}{l}\text { Oxycodone intrases- } \\
\text { sion analysis: day } 3\end{array}$ & LMM & $\begin{array}{c}\text { Genotype } \times \\
\text { timebin }\end{array}$ & N.S. & N.A. & N.S., $n=9$ & N.S., $n=8$ & N.S., $n=11$ & N.S., $n=9$ \\
\hline p & $3 K$ & $\begin{array}{l}\text { Oxycodone intrases- } \\
\text { sion analysis: day } 3\end{array}$ & LMM & Timebin & $\begin{array}{r}\chi^{2}=12.66 \\
p=0.027\end{array}$ & N.S., $n=11$ & $p<0.001, n=9$ & N.S., $n=8$ & N.S., $n=11$ & N.S., $n=9$ \\
\hline q & $3 L$ & $\begin{array}{l}\text { Morphine intrasession } \\
\text { analysis: day } 1\end{array}$ & LMM & $\begin{array}{c}\text { Genotype } \times \\
\text { timebin }\end{array}$ & $\begin{array}{r}\chi^{2}=21.239 \\
p<0.001\end{array}$ & N.A. & $p=0.002$ & N.S. & N.S. & N.S. \\
\hline $\mathbf{r}$ & $3 L$ & $\begin{array}{l}\text { Morphine intrasession } \\
\text { analysis: day } 1\end{array}$ & LMM & Timebin & $\begin{aligned} \chi^{2} & =54.796 \\
& p<0.0001\end{aligned}$ & $p<0.0001$ & $\begin{array}{l}\text { N.S., } \\
\qquad p=0.79\end{array}$ & $p<0.0001$ & $p<0.0001$ & $p=0.002$ \\
\hline $\mathbf{s}$ & $3 M$ & $\begin{array}{l}\text { Morphine intrasession } \\
\text { analysis: day } 3\end{array}$ & LMM & $\begin{array}{c}\text { Genotype } \times \\
\text { timebin }\end{array}$ & $\begin{aligned} \chi^{2} & =32.962 \\
p & <0.0001\end{aligned}$ & N.A. & $p<0.0001$ & N.S. & N.S. & $p<0.0001$ \\
\hline$t$ & $3 M$ & $\begin{array}{l}\text { Morphine intrasession } \\
\text { analysis: day } 3\end{array}$ & LMM & Timebin & $\begin{aligned} \chi^{2} & =64.194 \\
p & <0.0001\end{aligned}$ & $p<0.0001$ & $\begin{array}{l}\text { N.S., } \\
\qquad p=0.56\end{array}$ & $p<0.0001$ & $p<0.0001$ & $p=0.03$ \\
\hline
\end{tabular}

N.S.: not significant, N.A: not applicable. 
A

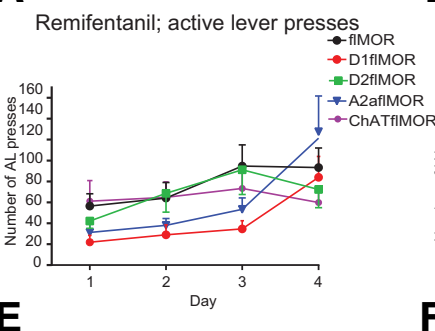

E

Oxycodone; active lever presse
B

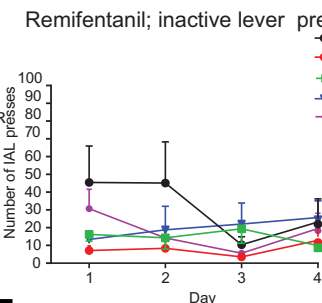

$\mathbf{F}$

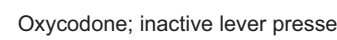

C

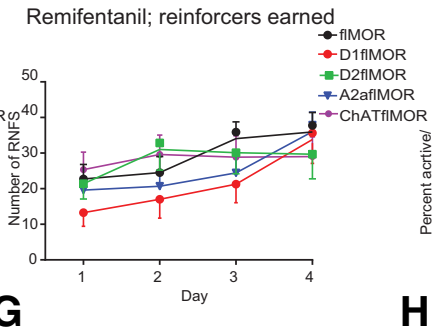

D
G

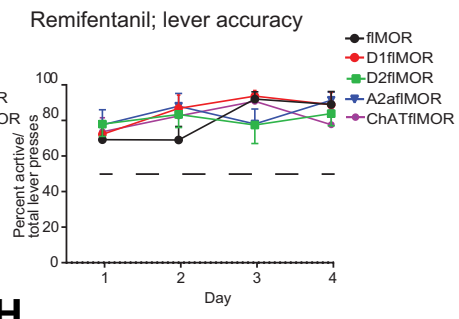

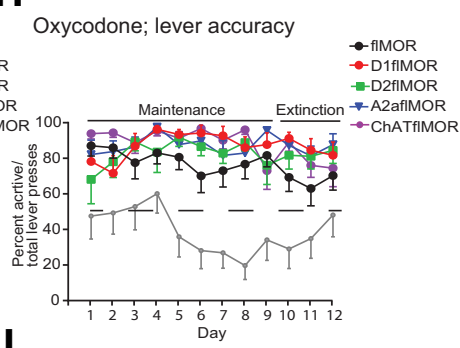

I

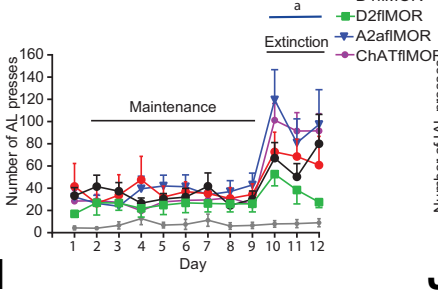

Last day oxycodone; active leve presses — FIMOR

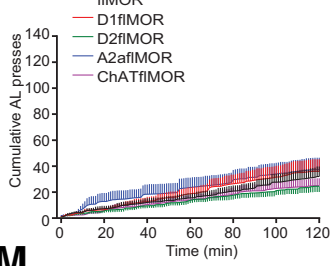

M

Last day oxycodone; reinforcers earned
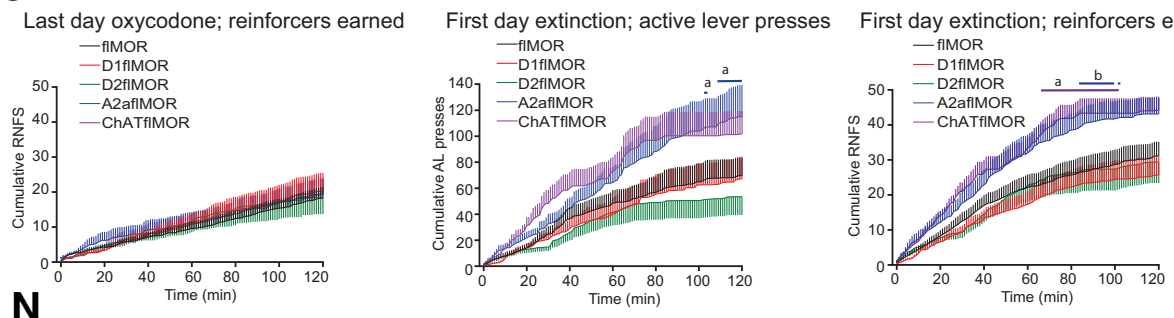

Third day extinction; active lever presses

Third day extinction; reinforcers earned
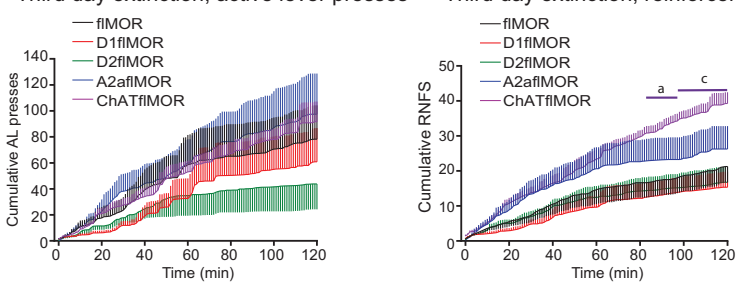

Figure 4. Selective MOR deletions define specific roles of A2a and ChAT populations in an opioid self-administration profile. $\boldsymbol{A}-\boldsymbol{D}$, Acquisition. During this short acquisition phase (days 1-4) during which remifentanil was self-administered there was no effect of genotype and no interaction or a main effect of genotype on any of the four parameters measured; active lever presses $(\boldsymbol{A})$, inactive lever presses $(\boldsymbol{B})$, reinforcers earned $(\boldsymbol{C})$, or the percent active lever presses made $(\boldsymbol{D})$. $\boldsymbol{E}-\boldsymbol{H}$, Maintenance and extinction. Mice were then transitioned to oxycodone self-administration for $9 \mathrm{~d}$ followed by $3 \mathrm{~d}$ of extinction. When compared with mice self-administering saline, those that self-administered oxycodone made more active lever presses $(p<0.05)$, earned more reinforcers $(p<0.05)$, and showed a preference for the active over inactive lever $(p<0.0001)$ during the maintenance and extinction session. $\boldsymbol{E}$, During the extinction but not maintenance phases, A2afIMORs made more active lever presses than fIMORs $(\boldsymbol{p}<0.05)$. $\boldsymbol{F}$, There was no effect of genotype on the number of inactive lever presses at any stage. G, Similar to the number of active lever presses made, A2afIMORs and ChATfIMORs earned more reinforcers than fIMORs during extinction (a: $p<0.05)$. $\boldsymbol{H}$, There was no effect of genotype on active lever preference as shown by the percent active lever/total lever presses. $\boldsymbol{I}-\boldsymbol{N}$, Within session analysis of the cumulative number of active lever presses made and reinforcers earned during the 2-h session was assessed on three specific days; the last day of oxycodone (day 9) and the first (day 10) and third (day 12) days of extinction. This shows no effect of genotype on the last day of oxycodone for either the cumulative active lever presses $(\boldsymbol{I})$ or reinforcers $(\boldsymbol{J})$ earned. $\boldsymbol{K}$, However, on the first day of extinction, A2afIMORs made more active lever presses than fIMORs (a: $p<0.05$ vs fIMOR at 103 and 104 and $110-120$ min). $I$, A similar effect was seen in the reinforcers earned during this session when A2afIMORs earned more reinforcers (a: $p<0.05$ vs fIMOR at 87-99 and $103 \mathrm{~min}$ ) as did ChATfIMORs (b: $p<0.05$ vs fIMOR at 69-102 min). $\boldsymbol{N}$, On the third day of extinction, there was no further effect of genotype on the number of active lever presses made. $\boldsymbol{M}$, However, the ChATfIMORs showed an increase in reinforcers earned on the third day of extinction (a and c: $p<0.05$ and $p<0.01$, respectively, vs fIMOR at $82-120$ min). Refer to Table 5 for statistical analyses. All data are shown as mean \pm SEM.

versus the last day of maintenance. No such transition effect was observed across any parameter in the saline group.

We then assessed the change in drug-seeking behavior over the $3 \mathrm{~d}$ of extinction in mice that had received oxycodone using LMM analysis. We found no genotype $\times$ day interaction, however there was a main effect of genotype on reinforcers earned (Fig. 4G, $p<0.05$; Table 5, item n) with ChATfIMORs $(p<0.01)$ and A2afIMORs $(p<0.05)$ earning more reinforcers than fIMOR mice over these $3 \mathrm{~d}$. There was a trend toward a main effect of genotype for 
Table 5: Statistical analyses of the IVSA profile in all lines (Fig. 4)

\begin{tabular}{|c|c|c|c|c|c|c|c|c|c|c|}
\hline Item & Figure & Experiment & Statistical test & Effect or interaction & Main effect & fIMOR & D1fIMOR & D2flMOR & A2aflMOR & ChATfIMOR \\
\hline $\mathbf{a}$ & $3 A$ & $\begin{array}{l}\text { Remifentanil } \\
\text { acquisition }\end{array}$ & LMM & AL day effect & $\begin{array}{l}p<0.0001, \\
\chi^{2}=21.017\end{array}$ & $n=15$ & $n=12$ & $n=10$ & $n=12$ & $n=12$ \\
\hline b & $3 C$ & $\begin{array}{l}\text { Remifentanil } \\
\text { acquisition }\end{array}$ & LMM & RNFS earned day effect & $\begin{array}{l}p<0.0001 \\
\chi^{2}=19.132\end{array}$ & & & & & \\
\hline c & $3 D$ & $\begin{array}{l}\text { Remifentanil } \\
\text { acquisition }\end{array}$ & LMM & Percent AL presses day effect & $\begin{array}{l}p<0.01 \\
x^{2}=9.730\end{array}$ & & & & & \\
\hline d & $3 E$ & $\begin{array}{l}\text { Oxycodone } \\
\text { maintenance }\end{array}$ & LMM & $\begin{array}{l}\text { AL presses } \times \text { Treatment } \\
\text { Oxycodone vs. Saline }\end{array}$ & $\begin{array}{l}p<0.001, \\
\quad \chi^{2}=10.926\end{array}$ & $\begin{array}{r}p<0.001, \chi^{2}= \\
11.601, n=14\end{array}$ & $\begin{array}{r}p<0.01, \chi^{2}= \\
6.764, n=9\end{array}$ & $\begin{array}{r}p<0.001, \chi^{2}= \\
12.806, n=9\end{array}$ & $\begin{aligned} p<0.01, \chi^{2} & = \\
10.545, n & =8\end{aligned}$ & $\begin{array}{c}p<0.001, \chi^{2}= \\
13.68, n=7\end{array}$ \\
\hline e & $3 G$ & $\begin{array}{l}\text { Oxycodone } \\
\text { maintenance }\end{array}$ & LMM & $\begin{array}{l}\text { RNFS } \times \text { treatment } \\
\text { Oxycodone vs. Saline }\end{array}$ & $\begin{array}{l}p<0.0001 \\
\quad \chi^{2}=16.051\end{array}$ & $\begin{array}{c}p<0.001, \chi^{2}= \\
\quad 11.435\end{array}$ & $\begin{array}{l}p<0.01, \chi^{2}= \\
\quad 8.434\end{array}$ & $\begin{array}{c}p<0.01, \chi^{2}= \\
9.339\end{array}$ & $\begin{array}{c}p<0.001, \chi^{2}= \\
10.981\end{array}$ & $\begin{array}{c}p<0.001, \chi^{2}= \\
14.648\end{array}$ \\
\hline f & $3 G$ & $\begin{array}{l}\text { Oxycodone } \\
\text { maintenance }\end{array}$ & LMM & $\begin{array}{l}\text { Percent AL presses treatment } \times \\
\text { day oxycodone vs saline }\end{array}$ & $\begin{array}{c}p<0.0001 \\
\quad \chi^{2}=34.81\end{array}$ & $\begin{array}{l}p<0.0001, \chi^{2}= \\
15.957\end{array}$ & $\begin{array}{l}p<0.0001, \chi^{2}= \\
\quad 20.334\end{array}$ & $\begin{array}{c}p<0.01, \chi^{2}= \\
7.918\end{array}$ & $\begin{array}{l}p<0.0001, \chi^{2}= \\
\quad 16.952\end{array}$ & $\begin{array}{l}p<0.0001, \chi^{2}= \\
19.941\end{array}$ \\
\hline g & $3 E$ & $\begin{array}{l}\text { Extinction; } \\
\text { transition }\end{array}$ & LMM & $\begin{array}{l}\text { AL presses treatment } \times \text { day } \\
\text { oxycodone vs. saline }\end{array}$ & $\begin{array}{l}p<0.01 \\
\quad \chi^{2}=8.296\end{array}$ & & & & & \\
\hline $\mathbf{h}$ & $3 G$ & $\begin{array}{l}\text { Extinction; } \\
\quad \text { transition }\end{array}$ & LMM & $\begin{array}{l}\text { RNFS treatment } \times \text { day } \\
\text { oxycodone vs. saline }\end{array}$ & $\begin{array}{l}p<0.01 \\
\chi^{2}=7.047\end{array}$ & & & & & \\
\hline i & $3 E$ & $\begin{array}{l}\text { Extinction; } \\
\quad \text { transition }\end{array}$ & LMM & $\begin{array}{l}\text { AL presses } \\
\text { day effect within } \\
\text { oxycodone treated }\end{array}$ & $\begin{array}{l}p<0.0001, \\
\chi^{2}=29.255\end{array}$ & & & & & \\
\hline j & $3 F$ & $\begin{array}{l}\text { Extinction; } \\
\quad \text { transition }\end{array}$ & LMM & $\begin{array}{l}\text { IAL presses } \\
\text { day effect within } \\
\text { oxycodone treated }\end{array}$ & $\begin{array}{l}p<0.01 \\
\chi^{2}=9.396\end{array}$ & & & & & \\
\hline k & $3 G$ & $\begin{array}{l}\text { Extinction; } \\
\text { transition }\end{array}$ & LMM & $\begin{array}{l}\text { RNFS day effect within } \\
\text { oxycodone treated }\end{array}$ & $\begin{array}{l}p<0.0001, \\
\chi^{2}=25.725\end{array}$ & & & & & \\
\hline 1 & $3 G$ & Extinction & LMM & $\begin{array}{l}\text { AL presses } \times \text { genotype } \\
\quad \text { within oxycodone treated }\end{array}$ & $\begin{aligned} p= & 0.050, \\
& \chi^{2}=9.480\end{aligned}$ & N.A. & N.S. & N.S. & $p=0.043$ & N.S. \\
\hline m & $3 G$ & Extinction & LMM & $\begin{array}{l}\text { IAL presses } \times \text { genotype } \\
\quad \text { within oxycodone treated }\end{array}$ & $\begin{array}{l}p=0.057 \\
\chi^{2}=9.148\end{array}$ & N.A. & N.S. & N.S. & $p=0.025$ & N.S. \\
\hline $\mathbf{n}$ & $3 G$ & Extinction & LMM & $\begin{array}{l}\text { RNFS earned } \times \text { genotype } \\
\quad \text { within oxycodone treated }\end{array}$ & $\begin{array}{l}p=0.018, \\
\chi^{2}=11.934\end{array}$ & N.A. & N.S. & N.S. & $p=0.031$ & $p=0.007$ \\
\hline o & $3 G$ & Extinction & LMM & $\begin{array}{r}\text { RNFS earned } \\
\text { day effect }\end{array}$ & $\begin{array}{l}p<0.001 \\
\quad \chi^{2}=12.979\end{array}$ & $\begin{array}{l}p<0.001 \\
t_{(50)}=-4.124\end{array}$ & $\begin{array}{l}p<0.001 \\
t_{(53)}=-3.964\end{array}$ & $\begin{array}{l}p<0.001 \\
t_{(55)}=-3.946\end{array}$ & $\begin{array}{l}p<0.001 \\
t_{(55)}=-3.135\end{array}$ & $\begin{array}{l}p<0.001 \\
t_{(56)}=-2.726\end{array}$ \\
\hline p & 31 & $\begin{array}{l}\text { Last day AL } \\
\text { oxycodone }\end{array}$ & $\begin{array}{l}\text { Two-way } \\
\text { ANOVA }\end{array}$ & Genotype $\times$ time & $\begin{array}{c}F_{(480,5160)}=0.45 \\
p>0.99\end{array}$ & & & & & \\
\hline q & $3 L$ & $\begin{array}{l}\text { Last day oxycodone } \\
\text { RNFS }\end{array}$ & $\begin{array}{l}\text { Two-way } \\
\text { ANOVA }\end{array}$ & Genotype $\times$ time & $\begin{array}{c}F_{(480,4920)}=0.2 \\
p>0.99\end{array}$ & & & & & \\
\hline $\mathbf{r}$ & $3 J$ & $\begin{array}{l}\text { First day } \\
\text { extinction } A L\end{array}$ & $\begin{array}{l}\text { Two-way } \\
\text { ANOVA }\end{array}$ & Genotype $\times$ time & $\begin{array}{c}F_{(480,4920)}=1.44 \\
p<0.0001\end{array}$ & $\begin{array}{l}\text { Reference } \\
\text { genotype }\end{array}$ & N.S. & N.S. & $p<0.05$ & N.S. \\
\hline s & $3 M$ & $\begin{array}{l}\text { First day } \\
\text { extinction RNFS }\end{array}$ & $\begin{array}{l}\text { Two-way } \\
\text { ANOVA }\end{array}$ & Genotype $\times$ time & $\begin{array}{c}F_{(480,4800)}=1.66 \\
p<0.0001\end{array}$ & $\begin{array}{l}\text { Reference } \\
\text { genotype }\end{array}$ & N.S. & N.S. & $p<0.05$ & $p<0.05$ \\
\hline$t$ & $3 \mathrm{~J}$ & $\begin{array}{l}\text { Third day } \\
\text { extinction AL }\end{array}$ & $\begin{array}{l}\text { Two-way } \\
\text { ANOVA }\end{array}$ & Genotype $\times$ time & $\begin{array}{c}F_{(480,5160)}=0.42 \\
p>0.99\end{array}$ & $\begin{array}{l}\text { Reference } \\
\text { genotype }\end{array}$ & N.S. & N.S. & N.S. & N.S. \\
\hline u & $3 M$ & $\begin{array}{l}\text { Third day } \\
\text { extinction RNFS }\end{array}$ & $\begin{array}{l}\text { Two-way } \\
\text { ANOVA }\end{array}$ & Genotype $\times$ time & $\begin{array}{c}F_{(480,5280)}=2.02 \\
p<0.0001\end{array}$ & $\begin{array}{l}\text { Reference } \\
\text { genotype }\end{array}$ & N.S. & N.S. & N.S. & $\begin{array}{c}p<0.05 \text { and } \\
\quad p<0.01\end{array}$ \\
\hline
\end{tabular}

N.S.: not significant, N.A: not applicable.

active lever presses (Fig. 3E, $p=0.0507$; Table 5, item I) and percentage active lever presses (Fig. $4 H, p=0.0571$; Table 5, item $\mathrm{m}$ ), with A2aflMORs showing increased active lever presses $(p<0.05)$ and percentage active lever presses $(p<0.05)$ made over these $3 d$ than the flMORs. We also observed a main effect of day on reinforcers earned (Fig. 4G, $p<0.0001$; Table 5, item o) with all mice showing a decrease in reinforcers earned over the $3 \mathrm{~d}$ of extinction with no effect of genotype. No other effects were found for active lever presses, inactive lever presses and percentage active lever presses across these $3 \mathrm{~d}$.

\section{Intrasession analysis}

We also analyzed the cumulative frequency of active lever presses and reinforcers earned during the 2 -h test on three specific days of the IVSA protocol (Fig. 4I-N). The first of these days, day 9 of the maintenance phase and the last day of oxycodone self-administration, showed a lack of genotype effect on either the cumulative active lever presses (Fig. 4/; Table 5, item p) or reinforcers earned (Fig. 4J; Table 5, item q). However, on the next day assessed, extinction day 1, A2aflMORS showed an increase in cumulative active lever presses (Fig. 4K, $p<0.05$; Table 5 , item $r$ ) and reinforcers earned (Fig. $4 L, p<0.05$; Table 5, item s). ChATfIMORs also earned more reinforcers than fIMORs on this day (Fig. $4 L$, $p<0.05$; Table 5 , item s). By the third day of extinction, there was no effect of genotype on cumulative active lever presses (Fig. 4M; Table 5, item $t$ ), but there was an effect of genotype on cumulative reinforcers earned with ChATfIMORs earning more reinforcers than flMORs during the last $40 \mathrm{~min}$ of the test (Fig. 4N; Table 5, item u). 


\section{Discussion}

These findings outline distinct roles for MORs on neuronal populations in behaviors associated with opioid-induced locomotion and reward behaviors. These are that selective ablation of MORs from D1 receptor-expressing neurons prevents opioid-induced locomotor hyperactivity as well as locomotor sensitization but has no effect on opioid IVSA. Second, removal of MORs from A2a neurons enhances opioid-induced hyperlocomotion, locomotor sensitization and drug-seeking behaviors during extinction following opioid IVSA. Third, ablation of MORs from ChAT neurons results in an agonist-dependent hyperlocomotor effect whereby morphine fails to elicit dose-dependent locomotor hyperactivity or sensitization yet oxycodone-induced effects are similar to control fIMOR mice. These mice also show an increase in drug-seeking behavior during extinction. Fourth, despite the common theory that A2a receptor expression is equivalent to D2 receptor expression in medium spiny neurons, our data suggests that the A2a cre deletes MORs from only a subset of D2 medium spiny neurons and, that, in stark contrast to MOR deletion from A2a neurons, MOR deletion from $\mathrm{D} 2$ neurons results in no discernible change in these reward-based behaviors (Fig. 5A).

Our study shows that MORs on D1 neurons are required for the initial locomotor and sensitization response to morphine and oxycodone. The effect of morphine is in line with a previous study in which the expression of MORs in only D1 neurons in striatal patches in an otherwise null background reinstated morphine-induced locomotion (Cui et al., 2014). Together these 2 findings demonstrate both the requirement and necessity of this MOR population for this striatal-mediated output. This may be a result of MORs on D1 recurrent collaterals inhibiting D2 neurons to reduce striatal output and attenuate the motor effect of opioids, as modeled in Figure $5 B$. Another possibility is that these receptors are required for the release of dopamine in the VTA (Cui et al., 2014), which is required for this response (Steidl et al., 2017). In regards our IVSA findings, the lack of effect of the D1 MOR deletion in the acquisition of oxycodone IVSA is in contrast with previous work (Cui et al., 2014), perhaps as other MOR populations such as those within the matrix, are also involved in the acquisition phase of this behavior. It is also possible that this is an example of an opioid-specific effect in which the faster-acting opioid, remifentanil, used in (Cui et al., 2014), results in greater lever pressing behavior than oxycodone.

As D2 receptors are expressed on cholinergic interneurons (Weiner et al., 1991), the A2a cre line has been used to selectively target D2 medium spiny neurons (Fink et al., 1992; Rosin et al., 2003; Wang et al., 2019). Our findings show that this A2a-MOR population is an apparent subset of D2 medium spiny neurons that controls the locomotor sensitivity to oxycodone and morphine and drug-seeking behavior during extinction. These inhibitory receptors may be on some D2-D1 collaterals (Taverna et al., 2008), where their deletion allows an earlier threshold to be reached to increase striatal motor output, as modeled in Figure 5B. As MORs on cholinergic interneurons remains intact and, surprisingly, MORs are also present on some D2 striatal neurons, their deletion displays a remarkably different and striking phenotype from D2flMORs. This could reflect a role of this striatal population or an extrastriatal neuronal population that expresses both $\mathrm{A} 2 \mathrm{a}$ and $\mu$ opioid but not necessarily D2 receptors. As regards MORs on D2 + neurons, we find that these receptors influence neither opioid-induced locomotion nor opioid IVSA.

While deleting MORS from D1, D2 and A2a neurons was performed to identify their role in GABAergic striatal neurons, deleting MORs from cholinergic interneurons examines the role of these receptors in altering cholinergic neuronal activity. These neurons form $1-3 \%$ of the striatal population yet they are remarkably influential in controlling striatal circuits (Gritton et al., 2019) and output, and both MORs and $\delta$-opioid receptors strongly inhibit their rhythmic activity to affect behavior (Bertran-Gonzalez et al., 2013; Ponterio et al., 2013). Activation of MORs could affect glutamate or acetylcholine release and subsequent dopamine release from nearby terminals (Yorgason et al., 2017 ) to alter the activity of local circuits (for review, see Clarke and Adermark, 2015; Berke, 2018). Omission of an expected reward induces a dip in dopamine release, a negative reward prediction error (RPE) accompanied by a pause in cholinergic interneuron activity (Hart et al., 2014) to affect local D1 and D2 medium spiny neuron activity (Mamaligas and Ford, 2016). Deleting MORs from these neurons may prevent the encoding of an RPE and facilitate drug-seeking, as shown by an increase in cue-induced reinforcers earned, but not active lever presses, during extinction (Fig. 4M,N).

The rapid increase in hyperlocomotion following oxycodone and the sustained, gradual increase in hyperlocomotion following morphine (Fig. $3 /-L$ ) is likely because of the different plasma-kinetic (PK) profiles of these two drugs. Oxycodone has a higher percentage of unbound drug in the blood and a 100-fold greater influx rate than morphine (Boström et al., 2008). This results in a 6-fold higher ratio of unbound oxycodone in the brain: blood and a higher unbound steady state in the brain (Boström et al., 2006, 2008) likely explaining the larger increase in dopamine release following intravenous oxycodone than intravenous morphine (Vander Weele et al., 2014). The ligand-dependent and genotype-dependent effect of morphine but not oxycodone in ChATfIMORs further suggests that this receptor population is more sensitive to the PK profile of each ligand. This could be because of a time-dependent effect of these receptors in modulating intrinsic cholinergic interneuron activity and the control of local circuitry.

There are several limitations of this study. One is that we have used the loxP-Cre recombinase system to achieve developmental deletion of MORs from various neuronal populations (Gong et al., 2007). For the most part these populations are striatal where the co-expression of MORs with D1 or D2 receptors can be used to define different medium spiny neuron populations (Gerfen et al., 1990; Weiner et al., 1991). However, dopamine neurons project to various brain regions in addition to the striatum, the hippocampus, amygdala, and prefrontal 
A

Oxycodone-induced effects

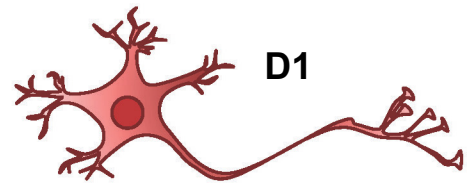

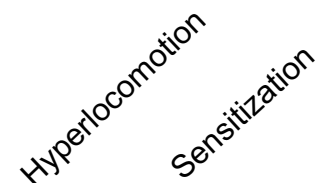

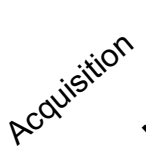<smiles>C#CC1CC1CC1CC1</smiles><smiles>[CH]1C=C1</smiles>

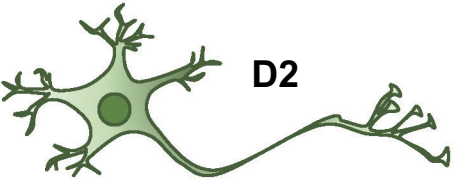<smiles>C1CC2CC1C2</smiles>
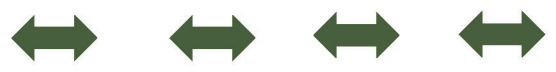

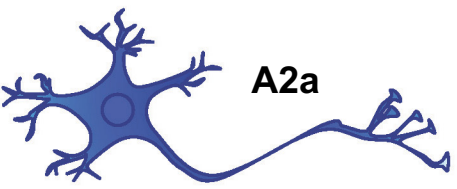
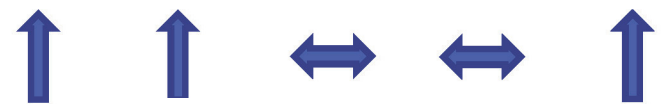

(reduced with morphine)
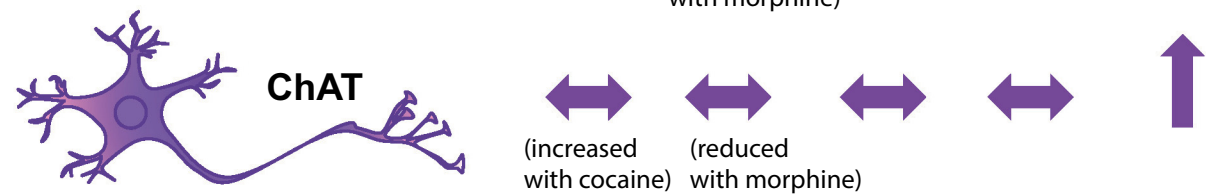

with cocaine) with morphine)

B

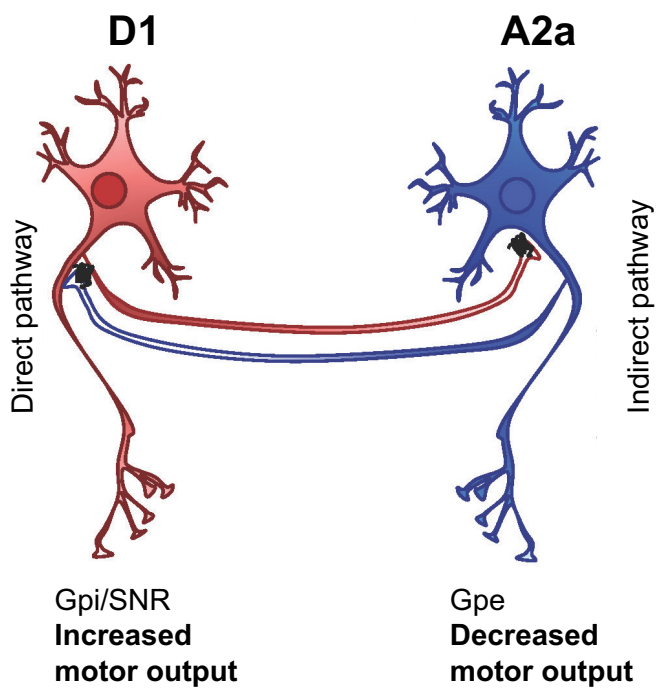

MORs are present on recurrent collaterals of GABAergic MSNs > disinbition of target MSNs.

Ablating MORs from D1 expressing neurons removes MOR inhibition and increases A2a MSN activity $>$ greater reduction in motor output.

Ablating MORs from A2a expressing neurons removes MOR inhibition and increases D1 MSN activity > greater increase in motor output.

Figure 5. A, Summary of our findings. Deleting MORs from D1 neurons reduces oxycodone-induced hyperlocomotion and sensitization but does not alter the IVSA profile. Deleting MORs from D2 neurons alters neither the locomotor effects of oxycodone nor the IVSA profile whereas deleting MORs from A2a neurons increases oxycodone-induced hyperlocomotion and sensitization and also drug-seeking behaviors following opioid IVSA. Deleting MORs from ChAT neurons does not alter oxycodone-induced hyperlocomotion and sensitization but does increase the locomotor effect of cocaine and drug-seeking behaviors following opioid IVSA. B, A possible mechanism by which MORs on D1 or A2a neurons alter striatal-mediated motor output. Removing MORs from D1 medium spiny neurons and so D1-A2a recurrent collateral increases A2a neuronal activity to reduce striatal motor output. Conversely removing MORs from A2a medium spiny neurons and so A2a-D1 recurrent collaterals increases D1 neuronal activity to increase striatal motor output.

cortex. The behavioral outcomes in this study may therefore be influenced by MOR expression on dopamine circuits outside the striatum. For example, MOR expression on the intercalated neurons of the amygdala (Gregoriou et al., 2019), and in the globus pallidus (Weiner et al., 1991; Delfs et al., 1994) may influence these reward-related behaviors. MOR expression on cholinergic neurons of the medial habenula (Gardon et al., 2014) may also influence 
reward behaviors (Boulos et al., 2020) and MORs and ChAT co-expression in secretomotor neurons of the colon suggests gut function may be altered in ChATfIMORs (Galligan and Akbarali, 2014). Further studies could also assess the role of MORs in different striatal subregions such as in patches or matrix, dorsal ventral striatum and co-expression with both D1 and D2 receptors (Soares-Cunha et al., 2016). An additional limitation is that we did not assess the effect of the cre insertion alone as this would have required further back-crossing of all lines.

Striatal D1 and D2 neurons are traditionally considered to have opposing effects on striatal motor patterns resulting in a coordinated motor activity. In this simple model, activating D1 neurons of the direct pathway increases striatal output to facilitate movement whereas activating D2 neurons of the indirect pathway inhibits competing motor patterns and inhibits movement (Kravitz et al., 2010). This model has been expanded and developed to include several interacting factors that influence the threshold of these outputs by recurrent collaterals between D1 and D2 neurons (Bahuguna et al., 2015), regulation by different interneurons (Taverna et al., 2008), and the regional and compartmental expression patterns of D1 and D2 (Cui et al., 2014; Oude Ophuis et al., 2014). Nevertheless, the opposing and complimentary effects of medium spiny neuron activation remains a central component of their activity. We show that the effect of deleting MORs from D1 and A2a neurons resembles such complementation, albeit the inverse, as it is the absence of MORs from D1 or A2a neurons that reduces or facilitates motor output, respectively. We propose that this can be explained by the presence of these $\mathrm{G}_{i 0}$-coupled receptors on recurrent medium spiny neuron collaterals, as shown by the schematic model in Figure $5 B$. The roles of D1 and D2 medium spiny neurons in mediating reward are also seen as divergent yet complementary in that D1 neurons mediate drug reinforcement and positive reward behaviors, whereas the D2s mediate aversion or ambivalence and are active during withdrawal (Koo et al., 2014; Cole et al., 2018). In addition, D1 and D2 receptors also play complementary but opposing roles in learning value-based and motivated behaviors, an important component of the change in reward value during extinction (Verharen et al., 2019). In regards the roles of MORs on these neurons, we show that rather than mediating positive reinforcement during the initial stages of opioid reward, that it is MORs on A2a or ChAT neurons that are important in controlling drug seeking during extinction, a period of increased anxiety and negative affect (Carmack et al., 2019). Additional studies to further define the effect of these deletions on A2a or ChAT neurons under different physiological conditions such as an increase in stress following periods of abstinence, or chronic pain, are needed to enhance our understanding of the complex and interrelated roles of these MOR populations.

\section{References}

Bahuguna J, Aertsen A, Kumar A (2015) Existence and control of Go/ No-Go decision transition threshold in the striatum. PLoS Comput Biol 11:e1004233.
Ben Hamida S, Boulos LJ, McNicholas M, Charbogne P, Kieffer BL (2017) Mu opioid receptors in GABAergic neurons of the forebrain promote alcohol reward and drinking. Addict Biol 24:28-39.

Berke JD (2018) What does dopamine mean? Nat Neurosci 21:787793.

Bertran-Gonzalez J, Laurent V, Chieng BC, Christie MJ, Balleine BW (2013) Learning-related translocation of $\delta$-opioid receptors on ventral striatal cholinergic interneurons mediates choice between goal-directed actions. J Neurosci 33:16060-16071.

Blomeley CP, Bracci E (2011) Opioidergic interactions between striatal projection neurons. J Neurosci 31:13346-13356.

Boström E, Simonsson US, Hammarlund-Udenaes M (2006) In vivo blood-brain barrier transport of oxycodone in the rat: indications for active influx and implications for pharmacokinetics/pharmacodynamics. Drug Metab Dispos 34:1624-1631.

Boström E, Hammarlund-Udenaes M, Simonsson US (2008) Bloodbrain barrier transport helps to explain discrepancies in in vivo potency between oxycodone and morphine. Anesthesiology 108:495505.

Boulos LJ, Nasseef MT, McNicholas M, Mechling A, Harsan LA, Darcq E, Ben Hamida S, Kieffer BL (2019) TouchScreen-based phenotyping: altered stimulus/reward association and lower perseveration to gain a reward in mu opioid receptor knockout mice. Sci Rep 9:4044.

Boulos LJ, Ben Hamida S, Bailly J, Maitra M, Ehrlich AT, Gavériaux-Ruff C, Darcq E, Kieffer BL (2020) Mu opioid receptors in the medial habenula contribute to naloxone aversion. Neuropsychopharmacology 45:247-255.

Brimblecombe KR, Cragg SJ (2017) The striosome and matrix compartments of the striatum: a path through the labyrinth from neurochemistry toward function. ACS Chem Neurosci 8:235-242.

Carmack SA, Keeley RJ, Vendruscolo JC, Lowery-Gionta EG, Lu H, Koob GF, Stein EA, Vendruscolo LF (2019) Heroin addiction engages negative emotional learning brain circuits in rats. J Clin Invest 129:2480-2484.

Charbogne P, Gardon O, Martín-García E, Keyworth HL, Matsui A, Mechling AE, Bienert T, Nasseef MT, Robé A, Moquin L, Darcq E, Ben Hamida S, Robledo P, Matifas A, Befort K, Gavériaux-Ruff C, Harsan LA, von Elverfeldt D, Hennig J, Gratton A, et al. (2017) Mu opioid receptors in gamma-aminobutyric acidergic forebrain neurons moderate motivation for heroin and palatable food. Biol Psychiatry 81:778-788.

Clarke R, Adermark L (2015) Dopaminergic regulation of striatal interneurons in reward and addiction: focus on alcohol. Neural Plast 2015:814567.

Cole SL, Robinson MJF, Berridge KC (2018) Optogenetic self-stimulation in the nucleus accumbens: D1 reward versus D2 ambivalence. PLoS One 13:e0207694.

Cui Y, Ostlund SB, James AS, Park CS, Ge W, Roberts KW, Mittal N, Murphy NP, Cepeda C, Kieffer BL, Levine MS, Jentsch JD, Walwyn WM, Sun YE, Evans CJ, Maidment NT, Yang XW (2014) Targeted expression of $\mu$-opioid receptors in a subset of striatal direct-pathway neurons restores opiate reward. Nat Neurosci 17:254-261.

Delfs JM, Kong H, Mestek A, Chen Y, Yu L, Reisine T, Chesselet MF (1994) Expression of mu opioid receptor mRNA in rat brain: an in situ hybridization study at the single cell level. J Comp Neurol 345:46-68.

Elghaba R, Bracci E (2017) Dichotomous effects of mu opioid receptor activation on striatal low-threshold spike interneurons. Front Cell Neurosci 11:385.

Everitt BJ, Giuliano C, Belin D (2018) Addictive behaviour in experimental animals: prospects for translation. Philos Trans R Soc Lond B Biol Sci 373:20170027.

Fink JS, Weaver DR, Rivkees SA, Peterfreund RA, Pollack AE, Adler EM, Reppert SM (1992) Molecular cloning of the rat A2 adenosine receptor: selective co-expression with $\mathrm{D} 2$ dopamine receptors in rat striatum. Brain Res Mol Brain Res 14:186-195.

Galligan JJ, Akbarali HI (2014) Molecular physiology of enteric opioid receptors. Am J Gastroenterol Suppl 2:17-21. 
Gardon O, Faget L, Chu Sin Chung P, Matifas A, Massotte D, Kieffer BL (2014) Expression of mu opioid receptor in dorsal diencephalic conduction system: new insights for the medial habenula. Neuroscience 277:595-609.

Gerfen CR, Engber TM, Mahan LC, Susel Z, Chase TN, Monsma FJ Jr, Sibley DR (1990) D1 and D2 dopamine receptor-regulated gene expression of striatonigral and striatopallidal neurons. Science 250:1429-1432.

Gong S, Doughty M, Harbaugh CR, Cummins A, Hatten ME, Heintz N, Gerfen CR (2007) Targeting Cre recombinase to specific neuron populations with bacterial artificial chromosome constructs. J Neurosci 27:9817-9823.

Gregoriou GC, Kissiwaa SA, Patel SD, Bagley EE (2019) Dopamine and opioids inhibit synaptic outputs of the main island of the intercalated neurons of the amygdala. Eur J Neurosci 50:2065-2074.

Gritton HJ, Howe WM, Romano MF, DiFeliceantonio AG, Kramer MA, Saligrama V, Bucklin ME, Zemel D, Han X (2019) Unique contributions of parvalbumin and cholinergic interneurons in organizing striatal networks during movement. Nat Neurosci 22:586-597.

Hakimian J, Minasyan A, Zhe-Ying L, Loureiro M, Beltrand A, Johnston C, Vorperian A, Romaneschi N, Atallah W, Gomez-Pinilla F, Walwyn W (2017) Specific behavioral and cellular adaptations induced by chronic morphine are reduced by dietary omega-3 polyunsaturated fatty acids. PLoS One 12:e0175090.

Hakimian JK, Dong TS, Barahona JA, Lagishetty V, Tiwari S, Azani D, Barrera M, Lee S, Severino AL, Mittal N, Cahill CM, Jacobs JP, Walwyn WM (2019) Dietary supplementation with omega-3 polyunsaturated fatty acids reduces opioid-seeking behaviors and alters the gut microbiome. Nutrients 11:1900.

Hart AS, Rutledge RB, Glimcher PW, Phillips PE (2014) Phasic dopamine release in the rat nucleus accumbens symmetrically encodes a reward prediction error term. J Neurosci 34:698-704.

Howe MW, Dombeck DA (2016) Rapid signalling in distinct dopaminergic axons during locomotion and reward. Nature 535:505-510.

James AS, Chen JY, Cepeda C, Mittal N, Jentsch JD, Levine MS, Evans CJ, Walwyn W (2013) Opioid self-administration results in cell-type specific adaptations of striatal medium spiny neurons. Behav Brain Res 256:279-283.

Koo JW, Lobo MK, Chaudhury D, Labonté B, Friedman A, Heller E, Pena CJ, Han MH, Nestler EJ (2014) Loss of BDNF signaling in D1R-expressing NAc neurons enhances morphine reward by reducing GABA inhibition. Neuropsychopharmacology 39:26462653.

Kravitz AV, Freeze BS, Parker PR, Kay K, Thwin MT, Deisseroth K, Kreitzer AC (2010) Regulation of parkinsonian motor behaviours by optogenetic control of basal ganglia circuitry. Nature 466:622626.

Kuznetsova A, Brockhoff PB, Christensen RHB (2017) ImerTest package: tests in linear mixed effects models. J Stat Softw 82:26.

Lawhorn C, Smith DM, Brown LL (2009) Partial ablation of mu-opioid receptor rich striosomes produces deficits on a motor-skill learning task. Neuroscience 163:109-119.

Le Merrer J, Becker JA, Befort K, Kieffer BL (2009) Reward processing by the opioid system in the brain. Physiol Rev 89:1379-1412.

Ma YY, Cepeda C, Chatta P, Franklin L, Evans CJ, Levine MS (2012) Regional and cell-type-specific effects of DAMGO on striatal D1 and D2 dopamine receptor-expressing medium-sized spiny neurons. ASN Neuro 4:AN20110063.

Mamaligas AA, Ford CP (2016) Spontaneous synaptic activation of muscarinic receptors by striatal cholinergic neuron firing. Neuron 91:574-586.

Mitchell JM, Cunningham CL, Mark GP (2005) Locomotor activity predicts acquisition of self-administration behavior but not cocaine intake. Behav Neurosci 119:464-472.

Mittal N, Minasyan A, Romaneschi N, Hakimian JK, GonzalezFernandez G, Albert R, Desai N, Mendez IA, Schallert T, Ostlund SB, Walwyn W (2017) Beta-arrestin 1 regulation of reward-motivated behaviors and glutamatergic function. PLoS One 12: e0185796.
Miura M, Masuda M, Aosaki T (2008) Roles of micro-opioid receptors in GABAergic synaptic transmission in the striosome and matrix compartments of the striatum. Mol Neurobiol 37:104-115.

Oude Ophuis RJ, Boender AJ, van Rozen AJ, Adan RA (2014) Cannabinoid, melanocortin and opioid receptor expression on DRD1 and DRD2 subpopulations in rat striatum. Front Neuroanat 8:14.

Ponterio G, Tassone A, Sciamanna G, Riahi E, Vanni V, Bonsi P, Pisani A (2013) Powerful inhibitory action of mu opioid receptors (MOR) on cholinergic interneuron excitability in the dorsal striatum. Neuropharmacology 75:78-85.

Ponterio G, Tassone A, Sciamanna G, Vanni V, Meringolo M, Santoro M, Mercuri NB, Bonsi P, Pisani A (2018) Enhanced mu opioid receptor-dependent opioidergic modulation of striatal cholinergic transmission in DYT1 dystonia. Mov Disord 33:310-320.

Robinson TE, Berridge KC (1993) The neural basis of drug craving: an incentive-sensitization theory of addiction. Brain Res Brain Res Rev 18:247-291.

Rosin DL, Hettinger BD, Lee A, Linden J (2003) Anatomy of adenosine A2A receptors in brain: morphological substrates for integration of striatal function. Neurology 61:S12-S18.

Sandor NT, Lendvai B, Vizi ES (1992) Effect of selective opiate antagonists on striatal acetylcholine and dopamine release. Brain Res Bull 29:369-373.

Severino A, Chen W, Hakimian JK, Kieffer BL, Gaveriaux-Ruff C, Walwyn W, Marvizón JCG (2018) Mu-opioid receptors in nociceptive afferents produce a sustained suppression of hyperalgesia in chronic pain. Pain 159:1607-1620.

Soares-Cunha C, Coimbra B, Sousa N, Rodrigues AJ (2016) Reappraising striatal D1- and D2-neurons in reward and aversion. Neurosci Biobehav Rev 68:370-386.

Steidl S, Wasserman DI, Blaha CD, Yeomans JS (2017) Opioid-induced rewards, locomotion, and dopamine activation: a proposed model for control by mesopontine and rostromedial tegmental neurons. Neurosci Biobehav Rev 83:72-82.

Stewart J, Badiani A (1993) Tolerance and sensitization to the behavioral effects of drugs. Behav Pharmacol 4:289-312.

Storey GP, Gonzalez-Fernandez G, Bamford IJ, Hur M, McKinley JW, Heimbigner L, Minasyan A, Walwyn WM, Bamford NS (2016) Nicotine modifies corticostriatal plasticity and amphetamine rewarding behaviors in mice $(1,2,3)$. eNeuro 3 .

Tao YM, Yu C, Wang WS, Hou YY, Xu XJ, Chi ZQ, Ding YQ, Wang YJ, Liu JG (2017) Heteromers of $\mu$ opioid and dopamine D1 receptors modulate opioid-induced locomotor sensitization in a dopamine-independent manner. Br J Pharmacol 174:2842-2861.

Taverna S, Ilijic E, Surmeier DJ (2008) Recurrent collateral connections of striatal medium spiny neurons are disrupted in models of Parkinson's disease. J Neurosci 28:5504-5512.

Vander Weele CM, Porter-Stransky KA, Mabrouk OS, Lovic V, Singer BF, Kennedy RT, Aragona BJ (2014) Rapid dopamine transmission within the nucleus accumbens: dramatic difference between morphine and oxycodone delivery. Eur J Neurosci 40:3041-3054.

Verharen JPH, Adan RAH, Vanderschuren L (2019) Differential contributions of striatal dopamine D1 and D2 receptors to component processes of value-based decision making. Neuropsychopharmacology 44:2195-2204.

Wang H, Pickel VM (1998) Dendritic spines containing $\mu$-opioid receptors in rat striatal patches receive asymmetric synapses from prefrontal corticostriatal afferents. J Comp Neurol 396:223-237.

Wang H, Moriwaki A, Wang JB, Uhl GR, Pickel VM (1996) Ultrastructural immunocytochemical localization of mu opioid receptors and Leu5-enkephalin in the patch compartment of the rat caudate-putamen nucleus. J Comp Neurol 375:659-674.

Wang H, Moriwaki A, Wang JB, Uhl GR, Pickel VM (1997) Ultrastructural immunocytochemical localization of mu-opioid receptors in dendritic targets of dopaminergic terminals in the rat caudate-putamen nucleus. Neuroscience 81:757-771.

Wang X, Qiao Y, Dai Z, Sui N, Shen F, Zhang J, Liang J (2019) Medium spiny neurons of the anterior dorsomedial striatum 
mediate reversal learning in a cell-type-dependent manner. Brain Struct Funct 224:419-434.

Weiner DM, Levey Al, Sunahara RK, Niznik HB, O'Dowd BF, Seeman P, Brann MR (1991) D1 and D2 dopamine receptor mRNA in rat brain. Proc Natl Acad Sci USA 88:1859-1863.

Yang PP, Yeh GC, Yeh TK, Xi J, Loh HH, Law PY, Tao PL (2016) Activation of delta-opioid receptor contributes to the antinociceptive effect of oxycodone in mice. Pharmacol Res 111:867-876.

Yorgason JT, Zeppenfeld DM, Williams JT (2017) Cholinergic interneurons underlie spontaneous dopamine release in nucleus accumbens. J Neurosci 37:2086-2096.

Zhang JJ, Kong Q (2017) Locomotor activity: a distinctive index in morphine self-administration in rats. PLoS One 12:e0174272. 